\title{
Mappings of open quantum systems onto chain representations and Markovian embeddings
}

\author{
M.P. Woods ${ }^{1,2}$, R. Groux ${ }^{3}$, A.W. Chin ${ }^{1}$, S.F. Huelga ${ }^{1}$, and M.B. Plenio ${ }^{1,2}$ \\ 1 Institute für Theoretische Physik, Universitatet Ulm, D-89069 Ulm, Germany \\ 2 QOLS, Blackett Laboratory, Imperial College London, SW7 2BW, United Kingdom \\ 3 Lycée Polyvalent Rouvière, Rue Sainte Claire Deville. BP 1205, 83070 Toulon, France \\ e-mail: mischa.woods05@imperial.ac.uk, roland.groux@orange.fr, alex.chin@uni-ulm.de, \\ susana.huelga@uni-ulm.de, martin.plenio@uni-ulm.de
}

Received: date / Accepted: date

\begin{abstract}
We derive a sequence of measures whose corresponding Jacobi matrices have special properties and a general mapping of an open quantum system onto 1D semi infinite chains with only nearest neighbour interactions. Then we proceed to use the sequence of measures and the properties of the Jacobi matrices to derive an expression for the spectral density describing the open quantum system when an increasing number of degrees of freedom in the environment have been embedded into the system. Finally, we derive convergence theorems for these residual spectral densities.
\end{abstract}

\section{Introduction}

\subsection{Background}

Real quantum mechanical systems are never found in complete isolation, but invariably coupled to a macroscopically large number of "environmental" degrees of freedom, such as those provided by electromagnetic field modes, density fluctuations of the surrounding media (phonons) or ensembles of other quantum systems, like electronic or nuclear spins [1,2,3]. The fact that the environment is totally or partially inaccessible to experimental probing in these so called "open quantum systems" leads to the appearance of an effectively irreversible dynamics for the quantum system's observables, and mediate the fundamental processes of energy relaxation, phase decoherence and, possibly, the thermalization of the subsystem.

Accurate numerical or analytical description of general open quantum systems dynamics appears, prima facie, to be extremely difficult due to the large (often infinite) number of bath and system variables which need to be accounted for. When the environmental degrees of freedom are modeled as a bath of harmonic oscillators exact path integral solutions can be available but are rarely of practical use 10,1,2. Hence assumptions such as weak 
system-environment coupling and vanishing correlation times of the environment, i.e. the Born-Markov approximation, are often invoked to obtain compact and efficiently solvable equations. These approaches suffer the drawback that their accuracy is hard to certify and that they become simply incorrect in many important situations. Indeed, our increasing ability to observe and control quantum systems on ever shorter time and length scales is constantly revealing new roles of noise and quantum coherence in important biological and chemical processes $[4,5,6,7,8$, and requires an accurate but efficient description of the system-environment interaction that go well beyond the Born-Markov approximation [8,9] in order to understand the interaction of intrinsic quantum dynamics and environmental noise. In many biological, chemical and solid-state systems, deviations from strict Markovianity, which can be explicitly quantified [11,12,13, are significant and methods beyond standard perturbative expansions are required for their efficient description. A number of techniques have been developed to operate in this regime. Those include polaron approaches [14, the quasi-adiabatic path-integral (QUAPI) method [15], the hierarchical equation of motion approach [16] and extensions of the quantum state diffusion description to nonMarkovian regimes [17].

Here we will focus on the exploration of the mathematics of an exact, analytical mapping of the standard model of a quantum system interacting with a continuum of harmonic oscillators to an equivalent model in which the system couples to one end of a chain nearest-neighbour coupled harmonic oscillators, as illustrated in (a) and (b) of Fig. (11). This mapping has permitted the formulation of an efficient algorithm for the description of the system-environment coupling for arbitrary spectral densities of the environment fluctuations [18, 19. This new mapping was originally intended just as a practical means of implementing t-DMRG which would avoid approximate determination of the chain representation using purely numerical, and often unstable, transforms [20. It was quickly realised that the scope of the mapping is much broader. Indeed, the mapping itself provides an extremely intuitive and powerful way of analysing universal properties of open quantum systems, that is, independent of the numerical method used to simulate the dynamics [18. This conclusion was implied also in [21], where the authors also developed a, in principle, rather different chain representation of a harmonic environment using an iterative propagator technique [21.

Both of these theories establish chain representations as a novel and direct way of looking at how energy and correlations propagate into the environment in real time. In the chain picture the interactions cause excitations to propagate away from the system, allowing a natural, causal understanding of Markovian and non-Markovian dissipation in terms of the properties of the chain's couplings and frequencies. An intuitive account of the physics of chain representations, non-Markovian dynamics and irreversibility for the method in [19. 18] is given in [22], and an interpretation of chain parameters in terms of time-correlations for the method in [21] can be found in [23].

\subsection{This communication}

The main goals of this paper can be split into two groups. Firstly, we aim at developing a general framework for mapping the environment of an open quantum system onto semi infinite 1D chain representations with nearest neighbour interactions where the system only couples to the first element in the chain. In these chain representations, there is a natural 
and systematic way to "embed" degrees of freedom of the environment into the system (by "embed", we mean to redefine what we call system and environment by including some of the environmental degrees of freedom in the system) (c) of figure (1). One can make a nonMarkovian system-environment interaction more Markovian by embedding some degrees of freedom of the environment into the system, a technique already employed in certain situations in quantum optics 24 and that recently has been demonstrated in 21. What remains unclear however, is to quantify how efficient such procedures can be as well as determining the best way of performing the embedding. We will show that those issues can be efficiently addressed with the formalism presented in this manuscript.

This general formalism allows the comparison between different chain mappings. Thus the second aim is to develop a method for understanding how Markovian such embeddings are by finding explicit analytical formulas for the spectral densities of the embeddings corresponding to the different chain mappings. Furthermore, we also derive universal convergence theorems for the spectral densities corresponding to the embedded systems and give rigourous conditions for when these limiting cases are achieved. This paves the way for a system interacting with a complex environment to be recast by moving the boundary of the system and environment, so that the non-trivial parts of the environment are embedded in the new effective "system" and the homogeneous chain represents the new, and much simpler, "environment" - See (c) in figure (1) for a pictorial illustration. The advantage of this is that the residual part of the environment might be simple enough for some of the approximations mentioned in section 1.1 to be applied, enabling us to integrate out these modes and dramatically reduce the number of sites of the chain that have to be accounted for explicitly. In order to achieve this, we have to first develop new mathematical tools and theorems regarding secondary measures and Jacobi operators, greatly extending and developing the application of orthogonal polynomials that was used in the original chain mapping of [18. These results might be useful also in other areas of mathematics and mathematical physics which are related to the theory of Jacobi operators such as the Toda lattice 25].

We show that this generalised chain mapping reduces to two known results mentioned in section 1.1 under special conditions. For the first known result [19, 18, the general method developed in this paper gives analytical and non-iterative expressions for the spectral densities corresponding to the embeddings. For the other special case 21, we derive calculable conditions for when the spectral densities corresponding to the embedding converge, -an aspect not addressed in [21]. As seen in the examples, we apply this technique to derive exact solutions for the family of Spin-Boson models which will allow us to illustrate how the different embedding methods are related. In addition, the method developed here is also valid when the spectral density of the system-enviroment interaction has a gap in its support region. This is of practical interest as there are open quantum systems (such as photonic crystals [26]) that naturally exhibit such a spectral density and hence can only be mapped onto a chain using the method presented in this paper.

The contents of each section is as follows: Section 2 is concerned with deriving the necessary mathematical tools for the application to open quantum systems in the subsequent sections. We start by introducing some elementary results in the field of orthogonal polynomials in section 2.1 (this section also helps to introduce notation). In section 2.2 we focus on deriving a formula which makes explicit a sequence of secondary measures solely in terms of the initial measure and its orthogonal polynomials. We point out that although authors such as Gautschi introduce the concept and definition of secondary measures, here 
we provide for the first time an analytical closed expression for them in terms of the initial measure and its orthogonal polynomials. This result will turn out to be a vital ingredient in the development of the subsequent chapters. Moreover, Gautschi states that the general solution we have found is unknown 27] p16-17. In section 2.3 we study the properties of the 3 -term recursion coefficients of the orthogonal polynomials generated from their measures. We then define beta normalised measures which have more general and useful properties. The main result of this section is our new theorem regarding the Jacobi matrix. This new theorem will be used extensively in section 3 . Section 3 is concerned with chain mappings for open quantum systems and embeddings of environmental degrees of freedom into the system. In section 3.1 we develop a general framework for mapping open quantum systems which are linearly coupled to an environment onto a representation where the environment is a semi-infinite chain with nearest neighbour couplings and define two special cases of particular interest, - the particle and phonon mappings. In section 3.2, we investigate the relation between this work and recent work by 21]. In order to do this we make extensive use of the relations developed in section 2. We show how their mapping is a special case of the work presented here and find analytical non iterative solutions to quantities such as the sequence of residual spectral densities. Section 3.3 is dedicated to deriving the formulas for the sequence of residual spectral densities for the particle case mapping. In section 3.4 we develop convergence theorems for the sequence of residual spectral densities. We show rigorously that the sequence converges under certain conditions and give the universal functions the spectral densities converge to for the particle and phonon cases. The conditions for which the sequences converge are stated in terms of the initial spectral density. In section 4 we give explicit analytic examples for the family of spectral densities of the Spin-Boson model for the particle and phonon mapping cases.

The main new results of this article are theorems (12), (15), (17), (18), (19), (20), (24) and corollaries (9) and (8).

\section{Secondary measures}

\subsection{Introduction to notation and basic tools}

Definition 1 Let us consider a measure $d \mu(x)=\bar{\mu}(x) d x$ with real support intervals $I_{1}, I_{2}, I_{3}, \ldots, I_{k}$ and $I=\left[\inf \left\{y, y \in I_{1} \cup I_{2} \cup I_{3} \cup \ldots \cup I_{k}\right\}, \sup \left\{y, y \in I_{1} \cup I_{2} \cup I_{3} \cup \ldots \cup I_{k}\right\}\right]=[a, b]$ with $a<b$, which has finite moments:

$$
C_{n}(d \mu)=\int_{a}^{b} x^{n} d \mu(x) \quad n=0,1,2, \ldots,
$$

with $\bar{\mu}(x) \geq 0$ over $I$.

We call $d \mu$ gapless measure if

$$
I=I_{1} \cup I_{2} \cup I_{3} \cup \ldots \cup I_{k}
$$

and gapped measure if

$$
I \neq I_{1} \cup I_{2} \cup I_{3} \cup \ldots \cup I_{k} .
$$

If not stated otherwise, a measure can be gapped or gapless and a, $b$ finite or infinite. 
Remark 1 The distinction between $a, b$ finite or infinite is important because as we will see, a few of the results in this paper (most noticeably, theorems 23 and 24), are restricted to the finite interval case. We also point out that another important classification of measures is that of gapped and gappless measures. Gapped measures also have much physical relevance and many of the theorems developed here apply equally well to these cases (see remark (7) for more details). It is also worth noting that in the case that $I$ is an unbounded interval, one has to be especially cautious when defining a measure, that all the moments are finite. Later on in this article (in proof to theorem [17), we will define measures as functions of spectral densities. Hence, for the case of spectral densities with unbounded support, this spectral density will only define a measure if the corresponding measure has finite moments. In practice, this is not really much of a restriction as the unbounded spectral densities found in the literature do have finite moments (such as in example 4.2).

As a final remark regarding measures, we note that the above definition is that of a positive measure. Some of the basic theorems and definitions of this section are also valid when the measure is not strictly positive. However, such cases are irrelevant for the new theorems developed here because a spectral density is a non-negative function. For this reason, we deal with only positive measures.

Definition 2 Let $\mathbb{P}$ denote the space of real polynomials. Then, for any $u(x)$ and $v(x) \in \mathbb{P}$ we will define an inner product as

$$
\langle u, v\rangle_{\bar{\mu}}=\int_{a}^{b} u(x) v(x) d \mu(x) .
$$

Definition 3 We call $\left\{P_{n}(d \mu ; x)\right\}_{n=0}^{\infty}$ the set of real orthonormal polynomials with respect to measure $d \mu$ where each polynomial $P_{n}$ is of degree $n$, if they satisfy

$$
\left\langle P_{n}(d \mu), P_{m}(d \mu)\right\rangle_{\bar{\mu}}=\delta_{n m} \quad n, m=0,1,2, \ldots .
$$

Similarly,

Definition 4 We call $\left\{\pi_{n}(d \mu ; x)\right\}_{n=0}^{\infty}$ the set of real monic polynomials with respect to measure $d \mu$ where each polynomial $\pi_{n}$ is of degree $n$ if they satisfy

$$
\pi_{n}(d \mu ; x)=P_{n}(d \mu ; x) / a_{n} \quad n=0,1,2, \ldots,
$$

where $a_{n}=a_{n}(d \mu)$ is the leading coefficient of $P_{n}(d \mu ; x)$.

Theorem 1 For any measure $d \mu(x)$, there always exists a set of real orthonormal polynomials and real monic polynomials.

Proof See [27.

Theorem 2 The monic polynomials $\pi_{n}(d \mu ; x)$ satisfy the three term recurrence relation

$$
\begin{gathered}
\pi_{n+1}(d \mu ; x)=\left(x-\alpha_{n}\right) \pi_{n}(d \mu ; x)-\beta_{n} \pi_{n-1}(d \mu ; x) \quad n=0,1,2, \ldots, \\
\pi_{-1}(d \mu ; x):=0,
\end{gathered}
$$


where

$$
\begin{aligned}
& \alpha_{n}=\alpha_{n}(d \mu)=\frac{\left\langle x \pi_{n}(d \mu), \pi_{n}(d \mu)\right\rangle_{\bar{\mu}}}{\left\langle\pi_{n}(d \mu), \pi_{n}(d \mu)\right\rangle_{\bar{\mu}}} \quad n=0,1,2, \ldots, \\
& \beta_{n}=\beta_{n}(d \mu)=\frac{\left\langle\pi_{n}(d \mu), \pi_{n}(d \mu)\right\rangle_{\bar{\mu}}}{\left\langle\pi_{n-1}(d \mu), \pi_{n-1}(d \mu)\right\rangle_{\bar{\mu}}} \quad n=1,2,3, \ldots .
\end{aligned}
$$

Proof See [27.

Definition 5 We will define $\beta_{0}(d \mu)$ by

$$
\beta_{0}(d \mu)=\left\langle\pi_{0}(d \mu), \pi_{0}(d \mu)\right\rangle_{\bar{\mu}} .
$$

Corollary $1 \beta_{0}(d \mu)=C_{0}(d \mu)$.

Proof We note that from definition (4),$\pi_{0}(d \mu ; x)=1$ for all measures $d \mu(x)$. Hence,

$$
\beta_{0}(d \mu)=\left\langle\pi_{0}(d \mu), \pi_{0}(d \mu)\right\rangle_{\bar{\mu}}=\int_{a}^{b} d \mu(x)=C_{0}(d \mu) .
$$

Theorem 3 When the measure $d \mu$ has bounded support, the $\alpha_{n}(d \mu)$ and $\beta_{n}(d \mu)$ coefficients are bounded by

$$
\begin{array}{cl}
a<\alpha_{n}(d \mu)<b & n=0,1,2, \ldots \\
0<\beta_{n}(d \mu) \leq \max \left(a^{2}, b^{2}\right) & n=0,1,2, \ldots
\end{array}
$$

Proof See [27.

Theorem 4 The orthonormal polynomials $P_{n}(d \mu ; x)$ satisfy the three term recurrence relation

$$
\begin{gathered}
t_{n} P_{n+1}(d \mu ; x)=\left(x-s_{n}\right) P_{n}(d \mu ; x)-t_{n-1} P_{n-1}(d \mu ; x) \quad n=0,1,2, \ldots, \\
P_{-1}(d \mu ; x):=0, \quad P_{0}(d \mu ; x)=1 / \sqrt{\beta_{0}(d \mu)},
\end{gathered}
$$

where

$$
\begin{aligned}
& s_{n}=s_{n}(d \mu)=\alpha_{n}(d \mu) \quad n=0,1,2, \ldots, \\
& t_{n}=t_{n}(d \mu)=\sqrt{\beta_{n+1}(d \mu)} \quad n=0,1,2, \ldots .
\end{aligned}
$$

Proof See [27.

Definition 6 We will call $Q_{n}(d \mu ; x)$ the secondary polynomia 11 associated with polynomial $P_{n}(d \mu ; x)$ defined by

$$
Q_{n}(d \mu ; x)=\int_{a}^{b} \frac{P_{n}(d \mu ; t)-P_{n}(d \mu ; x)}{t-x} d \mu(t), \quad n=0,1,2, \ldots
$$

\footnotetext{
${ }^{1}$ also known as polynomial of the second kind.
} 
Lemma 1 The polynomials $Q_{n}(d \mu ; x), n=1,2,3, \ldots$ are real polynomials of degree $n-1$ and $Q_{0}=0$.

Proof Follows from writing $P_{n}(d \mu ; x)$ in the form $P_{n}(d \mu ; x)=\sum_{q=0}^{n} k_{q} x^{q}$, using the identity $t^{q}-x^{q}=(t-x) \sum_{p=0}^{p=q-1} t^{p} x^{q-1-p}, \quad q=1,2,3, \ldots$ to cancel the denominator in Eq. (19) and noting that $C_{0}(d \mu)>0$.

Definition 7 The Stieltjes Transformation of the gapless measure $d \mu(x)=\bar{\mu}(x) d x$ is defined by 28

$$
S_{\bar{\mu}}(z)=\int_{a}^{b} \frac{d \mu(x)}{z-x}
$$

where $z \in \mathbb{C}-[a, b]$.

This is a function vanishing at infinity and analytic in the whole complex plane with the interval $[a, b]$ removed. (If $-a=b=+1$, then $S_{\bar{\mu}}$ is analytic separately in $\operatorname{Im} z>0$ and $\operatorname{Im}$ $z<0$, the two branches being different in general.)

Theorem 5 If a gapless measure $d \rho(x)=\rho(x) d x$ has Stieltjes transformation given by

$$
S_{\bar{\rho}}(z)=z-C_{1}(d \mu)-\frac{1}{S_{\bar{\mu}}(z)}
$$

with $C_{0}(d \mu)=1$, then the secondary polynomials $\left\{Q_{n}(d \mu ; x)\right\}_{n=1}^{\infty}$ form an orthogonal family for the induced inner product of $d \rho(x)$ and $d \mu(x)$ is also gapless.

Proof See 29], or [30] for a direct proof.

\subsection{Derivation of the sequence of secondary normalised measures}

Definition 8 For two gapless measures $d \rho(x)$ and $d \mu(x)$ satisfying Eq. (21), we call $d \rho(x)$ the secondary measure associated with $d \mu(x)$.

Definition 9 We call the sequence of gapless measures $d \mu_{0}, d \mu_{1}, d \mu_{2}, \ldots$ generated from a gapless measure $d \mu_{0}$ by

$$
\begin{aligned}
S_{\bar{\rho}_{n+1}}(z) & =z-C_{1}\left(d \mu_{n}\right)-\frac{1}{S_{\bar{\mu}_{n}}(z)} \quad n=0,1,2, \ldots, \\
d \mu_{n}(x) & =\bar{\mu}_{n}(x) d x \quad n=0,1,2, \ldots, \\
\bar{\mu}_{n}(x) & =\frac{\bar{\rho}_{n}(x)}{C_{0}\left(d \rho_{n}\right)} \quad n=1,2,3, \ldots,
\end{aligned}
$$

the sequence of normalised secondary measures, where $C_{0}\left(d \mu_{0}\right)=1$.

We note tha all sequences of normalised secondary measures are gapless by definition. This sequence of measures is a slight adaptation from a basic result in the theory of orthogonal polynomials. In the standard version, the sequence of measures are not normalised. The fact that these objects are actually positive measures, is a well known result of the basic theory. See 29] or [30. 
Corollary 2 All measures in a sequence of normalised secondary measures have their zeroth moment equal to unity.

Proof By taking zeroth moment of both sides of Eq. (24) we find

$$
C_{0}\left(d \mu_{n}\right)=\frac{C_{0}\left(d \rho_{n}\right)}{C_{0}\left(d \rho_{n}\right)}=1 \quad n=1,2,3, \ldots .
$$

We also have that $C_{0}\left(d \mu_{0}\right)=1$ by definition (9).

\section{Lemma 2}

$$
C_{n}\left(d \rho_{m+1}\right)=C_{n+2}\left(d \mu_{m}\right)-C_{1}\left(d \mu_{m}\right) C_{n+1}\left(d \mu_{m}\right)-\sum_{s=0}^{n-1} C_{s}\left(d \rho_{m+1}\right) C_{n-s}\left(d \mu_{m}\right)
$$

$n, m=0,1,2, \ldots$.

Proof For simplifity we will prove Eq. (26) for $m=0$ as the generalisation is trivial. By Taylor expanding $S_{\bar{\mu}_{0}}(z)$ and $S_{\bar{\rho}_{1}}(z)$ in $x=1 / z$ we find

$$
\begin{aligned}
& S_{\bar{\mu}_{0}}(z)=\sum_{n=0}^{\infty} \frac{C_{n}\left(d \mu_{0}\right)}{z^{n+1}} \quad \text { as } z \rightarrow \infty, \\
& S_{\bar{\rho}_{1}}(z)=\sum_{n=0}^{\infty} \frac{C_{n}\left(d \rho_{1}\right)}{z^{n+1}} \quad \text { as } z \rightarrow \infty .
\end{aligned}
$$

From Eq. (21) we have

$$
S_{\bar{\rho}_{1}}(z) S_{\bar{\mu}_{0}}(z)=\left(z-C_{1}\left(d \mu_{0}\right)\right) S_{\bar{\rho}_{1}}(z)-1 .
$$

Hence substituting Eq. (27) and (28) into Eq. (29) we find

$$
\sum_{n, m=0}^{\infty} C_{n}\left(d \mu_{0}\right) C_{m}\left(d \rho_{1}\right) x^{n+m+2}=\left(\frac{1}{x}-C_{1}\left(d \mu_{0}\right)\right) \sum_{s=0}^{\infty} C_{s}\left(d \mu_{0}\right) x^{s+1}-1 .
$$

By comparing terms of the same power in $x$ and taking into account $C_{0}\left(d \mu_{0}\right)=1$ we deduce

$$
\sum_{n=0}^{m} C_{n}\left(d \mu_{0}\right) C_{m-n}\left(d \rho_{1}\right)=C_{m+2}\left(d \mu_{0}\right)-C_{1}\left(d \mu_{0}\right) C_{m+1}\left(d \mu_{0}\right) \quad m=0,1,2, \ldots
$$

By a change of variable in Eq. (31) we finally arrive at Eq. (26).

Lemma 3 A sequence of normalised secondary measures $d \mu_{0}, d \mu_{1}, d \mu_{2}, \ldots, d \mu_{n}$, can be written as a continued fraction of the form

$$
S_{0}(z)=\frac{1}{z-C_{1,0}-\frac{d_{0}}{z-C_{1,1}-\frac{d_{1}}{z-C_{1,2}-\frac{d_{2}}{z-\cdots \frac{d_{n-1}}{z-C_{1, n-1}-\frac{d_{n+1}(z)}{z-C_{1, n}-d_{n} S_{n+1}}}}}}}
$$


where we have introduced the shorthand notation $S_{n}(z):=S_{\bar{\mu}_{n}}(z), C_{n, s}:=C_{n}\left(d \mu_{s}\right), \quad n, s=$ $0,1,2, \ldots ; d_{n}:=C_{2, n}-C_{1, n}^{2}, \quad n=1,2,3, \ldots$, and $d_{0}:=1$.

Proof By evaluating Eq. (26) for $n=0$, and taking into account the above definition of $d_{n}$ we see that $d_{n}=C\left(d \rho_{n}\right), n=1,2,3, \ldots$ Using our new notation, Eq. (22) for the sequence of gapless measures reads

$$
S_{n+1}(z)=\frac{1}{d_{n}}\left[z-C_{1, n}-\frac{1}{S_{n}(z)}\right], \quad n=0,1,2, \ldots .
$$

Solving this for $S_{n}(z)$ followed by repeated substitution gives us Eq. (32).

Theorem 6 The following relations hold for the continued fraction Eq. (32)

$$
S_{0}(z)=\frac{u_{n}\left(-d_{n} S_{n+1}(z)\right)+u_{n+1}}{v_{n}\left(-d_{n} S_{n+1}(z)\right)+v_{n+1}}, \quad n=0,1,2, \ldots,
$$

with relations

$$
u_{n+1}=\left(z-C_{1, n}\right) u_{n}-d_{n-1} u_{n-1}, \quad v_{n+1}=\left(z-C_{1, n}\right) v_{n}-d_{n-1} v_{n-1},
$$

and starting values

$$
u_{0}=0, \quad u_{1}=1, \quad v_{0}=1, \quad v_{1}=z-C_{0,1} .
$$

Proof These are elementary results from the theory of continued fractions (e.g. see section 4 , connection with continued fractions [31]).

\section{Lemma 4}

$$
\begin{aligned}
\Delta_{n+1} & :=u_{n+1} v_{n}-v_{n+1} u_{n}=d_{0} d_{1} d_{2} \ldots d_{n-1}, \quad n=1,2,3, \ldots, \\
\Delta_{1} & =1 .
\end{aligned}
$$

Proof Using Eq. (35) to substitute for $u_{n+1}$ and $v_{n+1}$ into Eq. (37), we find the relation $\Delta_{n+1}=d_{n-1} \Delta_{n}$. Using Eq. (36) to verify Eq. (37) for the starting values, Eq. (37) follows by induction.

Definition 10 A Padé Approximant for a function $g$ of type $q / p$ in the neighbourhood of 0 is a rational fraction

$$
F(z)=\frac{Q(z)}{P(z)}
$$

with degree of $Q \leq q$, degree of $P \leq p$ and $g(z)-\frac{Q(z)}{P(z)}$ of order $\mathcal{O}\left(z^{p+q+1}\right)$ in the neighbourhood of 0 .

For more details, see [31].

Theorem $7 F_{n}(z)=\frac{u_{n+1}(z)}{v_{n+1}(z)}$ is a Padé Approximant for $S_{0}(z)$ of type $n /(n+1), \quad n=$ $0,1,2, \ldots$. 
Proof Using theorem (6), we can write $S_{0}(z)-\frac{u_{n+1}(z)}{v_{n+1}(z)}$ as

$$
S_{0}(z)-\frac{u_{n+1}(z)}{v_{n+1}(z)}=\frac{\Delta_{n+1} d_{n} S_{n+1}(z)}{v_{n+1}(z)\left(v_{n+1}(z)-d_{n} v_{n}(z) S_{n+1}(z)\right)}, \quad n=0,1,2, \ldots .
$$

Through lemma (4) we see that $\Delta_{n+1}$ is independent of $z$. By Taylor expanding $S_{n+1}(z)$ defined in definition (7) about $x=1 / z$, and remembering that $C_{0}\left(d \mu_{n}\right)=1 \quad n=1,2,3 \ldots$, we find using Eq. (40) that

$$
S_{n+1}(z)=\frac{1}{z}+\mathcal{O}\left(\frac{1}{z^{2}}\right) \quad \text { as } z \rightarrow \infty \quad n=0,1,2, \ldots
$$

By induction, we see that $v_{n+1}$ and $u_{n+1}, \quad n=0,1,2, \ldots$ given by Eq. (35) and (36), are degree $n+1$ and $n$ polynomials in $z$ respectively, both with leading coefficients equal to unity. Hence we conclude that

$$
S_{0}(z)-\frac{u_{n+1}(z)}{v_{n+1}(z)}=\frac{\Delta_{n+1} d_{n}}{z^{2 n+3}}=\frac{d_{0} d_{1} \ldots d_{n}}{z^{2 n+3}} \quad \text { as } z \rightarrow \infty \quad n=0,1,2, \ldots
$$

Thus by definition (10) we conclude the proof.

Lemma $5 u_{n}(z)=\lambda_{n} Q_{n}\left(d \mu_{0} ; z\right)$ and $v_{n}(z)=\lambda_{n} P_{n}\left(d \mu_{0} ; z\right) \quad n=0,1,2, \ldots$, with $\lambda_{n}=$ $1 / a_{n}$ were $a_{n}$ is defined in definition (4).

Proof From section 5.3.: moment problems and orthogonal polynomials (p213-220) of [31, and theorem (7) it follows that

$$
\frac{u_{n+1}(z)}{v_{n+1}(z)}=\frac{Q_{n+1}\left(d \mu_{0} ; z\right)}{P_{n+1}\left(d \mu_{0} ; z\right)} \quad n=0,1,2, \ldots .
$$

By observing the starting values, we also have that

$$
\frac{u_{0}(z)}{v_{0}(z)}=\frac{Q_{0}\left(d \mu_{0} ; z\right)}{P_{0}\left(d \mu_{0} ; z\right)}
$$

Hence

$$
u_{n}(z)=\lambda_{n} Q_{n}\left(d \mu_{0} ; z\right) \quad \text { and } \quad v_{n}(z)=\lambda_{n} P_{n}\left(d \mu_{0} ; z\right) \quad n=0,1,2, \ldots .
$$

Given that $u_{n}$ and $v_{n}$ have leading coefficients equal to 1 , we must have $\lambda_{n}=1 / a_{n}$ $\mathrm{n}=0,1,2, \ldots$.

Theorem $8 d_{n}=a_{n}^{2} / a_{n+1}^{2}=\beta_{n+1}\left(d \mu_{0}\right) \quad n=0,1,2, \ldots$.

Proof Proceeding in the same way as in page (18) of [27, we have

$$
S_{0}(z)-\frac{Q_{n}\left(d \mu_{0} ; z\right)}{P_{n}\left(d \mu_{0} ; z\right)}=\frac{\gamma_{n}}{z^{2 n+1}} \quad \text { as } z \rightarrow \infty \quad n=0,1,2, \ldots
$$

where

$$
\gamma_{n}=\frac{1}{a_{n}} \int_{a}^{b} x^{n} P_{n}\left(d \mu_{0} ; x\right) d \mu_{0}(x)=\frac{\gamma_{0} a_{0}^{2}}{a_{n}^{2}} \frac{\left\langle P_{n}\left(d \mu_{0}\right), P_{n}\left(d \mu_{0}\right)\right\rangle_{\bar{\mu}}}{\left\langle P_{0}\left(d \mu_{0}\right), P_{0}\left(d \mu_{0}\right)\right\rangle_{\bar{\mu}}} \quad n=0,1,2, \ldots .
$$


Noting that $P_{0}\left(d \mu_{0} ; x\right) / a_{0}=\pi_{0}\left(d \mu_{0} ; x\right)=1$ and that $C_{0}\left(d \mu_{0}\right)=1$, Eq. (47) tells us $\gamma_{0}=1$. Comparing Eq. (46) with Eq. (42) and (43), we deduce that

$$
d_{0} d_{1} \ldots d_{n-1}=\frac{a_{0}^{2}}{a_{n}^{2}} \frac{\left\langle P_{n}\left(d \mu_{0}\right), P_{n}\left(d \mu_{0}\right)\right\rangle_{\bar{\mu}}}{\left\langle P_{0}\left(d \mu_{0}\right), P_{0}\left(d \mu_{0}\right)\right\rangle_{\bar{\mu}}} \quad n=1,2,3, \ldots
$$

By induction it follows

$$
d_{n}=\frac{a_{n}^{2}}{a_{n+1}^{2}} \frac{\left\langle P_{n+1}\left(d \mu_{0}\right), P_{n+1}\left(d \mu_{0}\right)\right\rangle_{\bar{\mu}}}{\left\langle P_{n}\left(d \mu_{0}\right), P_{n}\left(d \mu_{0}\right)\right\rangle_{\bar{\mu}}} \quad n=1,2,3, \ldots .
$$

Due to definition (3), we see that $\left\langle P_{n}\left(d \mu_{0}\right), P_{n}\left(d \mu_{0}\right)\right\rangle_{\bar{\mu}}=1 \quad n=0,1,2, \ldots$, hence

$$
d_{n}=\frac{a_{n}^{2}}{a_{n+1}^{2}} \quad n=1,2,3, \ldots
$$

From definition (4), we see that Eq. (49) can be written in the form

$$
d_{n}=\frac{\left\langle\pi_{n+1}\left(d \mu_{0}\right), \pi_{n+1}\left(d \mu_{0}\right)\right\rangle_{\bar{\mu}}}{\left\langle\pi_{n}\left(d \mu_{0}\right), \pi_{n}\left(d \mu_{0}\right)\right\rangle_{\bar{\mu}}} \quad n=1,2,3, \ldots
$$

Hence, from definition (10) we conclude,

$$
d_{n}=\beta_{n+1}\left(d \mu_{0}\right) \quad n=1,2,3, \ldots .
$$

For $n=1$, Eq. (48) gives us

$$
d_{0}=\frac{\left\langle\pi_{1}\left(d \mu_{0}\right), \pi_{1}\left(d \mu_{0}\right)\right\rangle_{\bar{\mu}}}{\left\langle\pi_{0}\left(d \mu_{0}\right), \pi_{0}\left(d \mu_{0}\right)\right\rangle_{\bar{\mu}}}=\frac{a_{0}^{2}}{a_{1}^{2}}=\beta_{1}\left(d \mu_{0}\right) .
$$

Theorem 9 Gapless measures $d \mu(x)=\bar{\mu}(x) d x$ can be calculated from their Stieltjes transform by

$$
\bar{\mu}(x)=\frac{1}{2 \pi i} \lim _{\epsilon \rightarrow 0^{+}}\left[S_{\bar{\mu}}(x-i \epsilon)-S_{\bar{\mu}}(x+i \epsilon)\right] .
$$

Proof This result is known as the Stieltjes-Perron inversion formula. See [32] for more details or Example 2.50: Stieltjes-Perron inversion formula [27] for an application and more references.

Definition 11 We call $\varphi(d \mu ; x)$ the reducer of gapless measure $d \mu(x)$. It is given by

$$
\varphi(d \mu ; x)=\lim _{\epsilon \rightarrow 0^{+}}\left[S_{\bar{\mu}}(x-i \epsilon)+S_{\bar{\mu}}(x+i \epsilon)\right] .
$$

See section 6.2 for methods for calculating the reducer. The reducer allows us to write an explicit expression for the secondary measure associated with $\bar{\mu}(x)$ as follows.

Theorem 10 For a gapless measure $d \mu(x)$ with secondary measure $d \rho(x)$, we have

$$
\bar{\rho}(x)=\frac{\bar{\mu}(x)}{\frac{\varphi^{2}(d \mu ; x)}{4}+\pi^{2} \bar{\mu}^{2}(x)}
$$


Proof See [30.

Definition 12 We define the functions $Z_{n}(x) \epsilon \mathbb{C} \quad n=0,1,2, \ldots$ as

$$
Z_{n}(x)=\frac{\varphi_{n}(x)}{2}+i \pi \bar{\mu}_{n}(x) \quad n=0,1,2, \ldots,
$$

where $\varphi_{n}(x):=\varphi\left(d \mu_{n} ; x\right)$.

Lemma 6 The following recursion relation hold for $Z_{n+1}(x)$

$$
Z_{n+1}(x)=\frac{1}{d_{n}}\left[x-C_{1, n}-\frac{1}{Z_{n}(x)}\right], \quad n=0,1,2, \ldots .
$$

Proof We start by finding a relation between the Stieltjes transformation of a gapless measure $d \mu(x)$, and the reducer of its associated secondary measure $d \rho(x)$ : By definition, we have

$$
\varphi(d \rho ; x)=\lim _{\epsilon \rightarrow 0^{+}}\left[S_{\bar{\rho}}(x-i \epsilon)+S_{\bar{\rho}}(x+i \epsilon)\right] .
$$

Using Eq. (21), we find

$$
\varphi(d \rho ; x)=2\left[x-C_{1}(d \mu)\right]-\lim _{\epsilon \rightarrow 0^{+}} \frac{S_{\bar{\mu}}(x-i \epsilon)+S_{\bar{\mu}}(x+i \epsilon)}{S_{\bar{\mu}}(x-i \epsilon) S_{\bar{\mu}}(x+i \epsilon)} .
$$

Now using theorem (9) and definition (11), we find that

$$
\lim _{\epsilon \rightarrow 0^{+}} \frac{S_{\bar{\mu}}(x-i \epsilon)+S_{\bar{\mu}}(x+i \epsilon)}{S_{\bar{\mu}}(x-i \epsilon) S_{\bar{\mu}}(x+i \epsilon)}=\frac{\varphi(d \mu ; x)}{\frac{\varphi^{2}(d \mu ; x)}{4}+\pi^{2} \bar{\mu}^{2}(x)} .
$$

Hence from Eq. (60) we arrive at

$$
\varphi(d \rho ; x)=2\left[x-C_{1}(d \mu)\right]-\frac{\varphi(d \mu ; x)}{\frac{\varphi^{2}(d \mu ; x)}{4}+\pi^{2} \bar{\mu}^{2}(x)} .
$$

Using the definition of a sequence of normalised secondary measures, definition (9) and the definition of $d_{n}$ in Lemma (3), from Eq. (62) we find

$$
\varphi_{n+1}(x)=\frac{1}{d_{n}}\left[2\left[x-C_{1, n}\right]-\frac{\varphi_{n}(x)}{\frac{\varphi_{n}^{2}(x)}{4}+\pi^{2} \bar{\mu}_{n}^{2}(x)}\right], \quad n=0,1,2, \ldots .
$$

Similarly, we can also write theorem (10) for our sequence of normalised secondary measures using definition (91) and $d_{n}$. We find

$$
\bar{\mu}_{n+1}(x)=\frac{1}{d_{n}}\left[\frac{\bar{\mu}_{n}(x)}{\frac{\varphi_{n}^{2}(x)}{4}+\pi^{2} \bar{\mu}_{n}^{2}(x)}\right], \quad n=0,1,2, \ldots .
$$

If we write Eq. (57) for $Z_{n+1}(x)$ and then substitute Eq. (63) and Eq. (64) into the RHS we arrive at Eq. (58). 
Theorem 11 The following relations hold between $Z_{0}(x)$ and $Z_{n+1}(x)$

$$
Z_{0}(z)=\frac{u_{n}\left(-d_{n} Z_{n+1}(z)\right)+u_{n+1}}{v_{n}\left(-d_{n} Z_{n+1}(z)\right)+v_{n+1}}, \quad n=0,1,2, \ldots,
$$

were $u_{n}(z)=\lambda_{n} Q_{n}\left(d \mu_{0} ; z\right)$ and $v_{n}(z)=\lambda_{n} P_{n}\left(d \mu_{0} ; z\right) \quad n=0,1,2, \ldots$, with $\lambda_{n}=1 / a_{n}, a_{n}$ defined in definition (4).

Proof By comparing Eq. (158) with eq. (33)), we note that $Z_{n}(x)$ satisfies the same recursion relation as $S_{n}(x)$. Hence theorem (34) readily applies to Eq. (58) if we exchange $S_{n+1}(x)$ with $Z_{n+1}(x)$ and $S_{0}(x)$ with $Z_{0}(x)$. The relations between $u_{n}, v_{n}$ and $Q_{n}, P_{n}$ are proven in lemma (5).

We are now ready to state our first main theorem:

Theorem $12 A$ sequence of normalised secondary measures starting from $d \mu_{0}: d \mu_{0}, d \mu_{1}$, $d \mu_{2}, \ldots, d \mu_{m}, \ldots$ can be generated from the first measure in the sequence $d \mu_{0}$ by the formula

$$
\bar{\mu}_{n}(x)=\frac{1}{t_{n-1}^{2}\left(d \mu_{0}\right)} \frac{\bar{\mu}_{0}(x)}{\left(P_{n-1}\left(d \mu_{0} ; x\right) \frac{\varphi\left(d \mu_{0} ; x\right)}{2}-Q_{n-1}\left(d \mu_{0} ; x\right)\right)^{2}+\pi^{2} \bar{\mu}_{0}^{2}(x) P_{n-1}^{2}\left(d \mu_{0} ; x\right)}
$$

$n=1,2,3, \ldots$,

where the $t_{n}$ coefficients are defined in theorem (4).

Proof After solving Eq. (65) for $Z_{n}(x)$, we find

$$
Z_{n}(x)=\frac{a_{n-1}}{a_{n} d_{n-1}} \frac{Z_{0}(x) P_{n}\left(d \mu_{0} ; x\right)-Q_{n}\left(d \mu_{0} ; x\right)}{Z_{0}(x) P_{n-1}\left(d \mu_{0} ; x\right)-Q_{n-1}\left(d \mu_{0} ; x\right)} \quad n=1,2,3, \ldots .
$$

From theorem (8) we see that

$$
a_{n-1} / a_{n}=\kappa_{n} \sqrt{d_{n-1}} \quad n=1,2,3, \ldots,
$$

where $\kappa_{n}$ is the sign of $a_{n-1} / a_{n}$. After using this relation to simplify the $a_{n-1} / a_{n} d_{n-1}$ coefficient in Eq. (67) and substituting for $Z_{0}(x)$ using definition (12), we take real and imaginary parts to achieve

$$
\bar{\mu}_{n}(x)=\frac{\kappa_{n}}{\sqrt{d_{n-1}}} \frac{\bar{\mu}_{0}(x)\left[P_{n-1}\left(d \mu_{0} ; x\right) Q_{n}\left(d \mu_{0} ; x\right)-P_{n}\left(d \mu_{0} ; x\right) Q_{n-1}\left(d \mu_{0} ; x\right)\right]}{\left(P_{n-1}\left(d \mu_{0} ; x\right) \frac{\varphi\left(d \mu_{0} ; x\right)}{2}-Q_{n-1}\left(d \mu_{0} ; x\right)\right)^{2}+\pi^{2} \bar{\mu}_{0}^{2}(x) P_{n-1}^{2}\left(d \mu_{0} ; x\right)}
$$

$n=1,2,3, \ldots$ Using the identities from lemmas (4) and (5), we note that

$$
P_{n}\left(d \mu_{0} ; x\right) Q_{n+1}\left(d \mu_{0} ; x\right)-P_{n+1}\left(d \mu_{0} ; x\right) Q_{n}\left(d \mu_{0} ; x\right)=d_{0} d_{1} \ldots d_{n-1} / \lambda_{n} \lambda_{n+1}
$$

$n=1,2,3, \ldots$. Using theorem (8) and lemma (5) to write the RHS in terms of the $a_{n}$ 's, we find

$$
d_{0} d_{1} \ldots d_{n-1} / \lambda_{n} \lambda_{n+1}=a_{0}^{2} a_{n+1} / a_{n}=a_{0}^{2} / \kappa_{n+1} \sqrt{d_{n}} \quad n=0,1,2, \ldots
$$


where in the last line we have used Eq. (68). We now note that by definition (4), $P_{0}\left(d \mu_{0} ; x\right)=$ $a_{0}$. We also have by definition (3), that $\left\langle P_{0}\left(d \mu_{0} ; x\right), P_{0}\left(d \mu_{0} ; x\right)\right\rangle_{\bar{\mu}_{0}}=1$. Hence

$$
1=\left\langle P_{0}\left(d \mu_{0} ; x\right), P_{0}\left(d \mu_{0} ; x\right)\right\rangle_{\bar{\mu}_{0}}=a_{0}^{2} C_{0}\left(d \mu_{0}\right)=a_{0}^{2} .
$$

Hence from Eq. (70), (171), (72), we find that we can write Eq. (69) as

$$
\bar{\mu}_{n}(x)=\frac{1}{d_{n-1}} \frac{\bar{\mu}_{0}(x)}{\left(P_{n-1}\left(d \mu_{0} ; x\right) \frac{\varphi\left(d \mu_{0} ; x\right)}{2}-Q_{n-1}\left(d \mu_{0} ; x\right)\right)^{2}+\pi^{2} \bar{\mu}_{0}^{2}(x) P_{n-1}^{2}\left(d \mu_{0} ; x\right)}
$$

$n=1,2,3, \ldots$. Finally, with the relations from theorems (8) and (44) we find $d_{n-1}=$ $t_{n-1}^{2}\left(d \mu_{0}\right) \quad n=1,2,3, \ldots$.

\subsection{Derivation of the Jacobi matrix theorem}

Lemma 7 The $\alpha_{n}(d \mu)$ and $\beta_{n}(d \mu)$ coefficients defined in theorem (2) are invariant under a change of scale of the measure $d \mu(x)$, while the change in $\beta_{0}(d \mu)$ scales linearly:

$$
\begin{aligned}
\alpha_{n}(C d \mu) & =\alpha_{n}(d \mu) \quad n=0,1,2, \ldots \\
\beta_{n}(C d \mu) & =\beta_{n}(d \mu) \quad n=1,2,3, \ldots \\
\beta_{0}(C d \mu) & =C \beta_{0}(d \mu)
\end{aligned}
$$

where $C>0$.

Proof First we will show that $\pi_{n}(C d \mu ; x)=\pi_{n}(d \mu ; x) \quad n=0,1,2, \ldots$

From definition (6), we have that

$$
1=\left\langle P_{n}(d \mu), P_{n}(d \mu)\right\rangle_{\bar{\mu}} \quad n=0,1,2, \ldots
$$

By multiplying and dividing by $C$ we find

$$
1=\left\langle\frac{P_{n}(d \mu)}{\sqrt{C}}, \frac{P_{n}(d \mu)}{\sqrt{C}}\right\rangle_{\overline{c \mu}} \quad n=0,1,2, \ldots
$$

From definition (6), we conclude

$$
P_{n}(C d \mu ; x)=\frac{P_{n}(d \mu ; x)}{\sqrt{C}} \quad n=0,1,2, \ldots
$$

By observing definition (44) and Eq. (79), we conclude

$$
a_{n}(C d \mu)=a_{n}(d \mu) / \sqrt{C} \quad n=0,1,2, \ldots .
$$

Hence, from Eq. (79), (80) and definition (6) it follows

$$
\pi_{n}(C d \mu ; x)=P_{n}(C d \mu ; x) / a_{n}(C d \mu)=P_{n}(d \mu ; x) / a_{n}(d \mu)=\pi_{n}(d \mu ; x)
$$


$n=0,1,2, \ldots$. Now we can see how the $\alpha_{n}$ and $\beta_{n}$ coefficients change:

Using definition (2) and Eq. (81) we have

$$
\begin{aligned}
\alpha_{n}(C d \mu) & =\frac{\left\langle x \pi_{n}(C d \mu), \pi_{n}(C d \mu)\right\rangle_{\overline{c \mu}}}{\left\langle\pi_{n}(C d \mu), \pi_{n}(C d \mu)\right\rangle_{\overline{c \mu}}} \\
& =\frac{\left\langle x \pi_{n}(d \mu), \pi_{n}(d \mu)\right\rangle_{\bar{\mu}}}{\left\langle\pi_{n}(d \mu), \pi_{n}(d \mu)\right\rangle_{\bar{\mu}}} \\
& =\alpha_{n}(d \mu), \quad n=0,1,2, \ldots
\end{aligned}
$$

Similarly, we find

$$
\beta_{n}(C d \mu)=\beta_{n}(d \mu), \quad n=1,2,3, \ldots .
$$

In the case of $\beta_{0}$ we have

$$
\beta_{0}(C d \mu)=\left\langle\pi_{0}(C d \mu), \pi_{0}(C d \mu)\right\rangle_{c \mu}=C \beta_{0}(d \mu) .
$$

Theorem 13 If $d \rho(x)$ is the secondary measure associated with $d \mu(x)$, then

$$
\begin{array}{ll}
\alpha_{n+1}(d \mu)=\alpha_{n}(d \rho) & n=0,1,2, \ldots \\
\beta_{n+1}(d \mu)=\beta_{n}(d \rho) & n=1,2,3, \ldots
\end{array}
$$

Proof See [27, theorem 1.36 (page 16).

Lemma 8 A sequence of normalised secondary measures $d \mu_{0}(x), d \mu_{1}(x), d \mu_{2}(x), \ldots$, satisfy

$$
\begin{array}{ll}
\alpha_{m+n}\left(d \mu_{0}\right)=\alpha_{n}\left(d \mu_{m}\right) & n, m=0,1,2, \ldots, \\
\beta_{m+n}\left(d \mu_{0}\right)=\beta_{n}\left(d \mu_{m}\right) & n=1,2,3, \ldots, m=0,1,2, \ldots .
\end{array}
$$

Proof Using lemma (7) and definition (9) we find

$$
\begin{aligned}
& \alpha_{n}\left(d \rho_{m}\right)=\alpha_{n}\left(d \rho_{m} / C_{0}\left(d \rho_{m}\right)\right)=\alpha_{n}\left(d \mu_{m}\right) \quad n=0,1,2, \ldots m=1,2,3 \ldots \\
& \beta_{n}\left(d \rho_{m}\right)=\beta_{n}\left(d \rho_{m} / C_{0}\left(d \rho_{m}\right)\right)=\beta_{n}\left(d \mu_{m}\right) \quad n=1,2,3, \ldots m=1,2,3 \ldots
\end{aligned}
$$

Hence taking into account theorem (13) we find

$$
\begin{array}{ll}
\alpha_{n+1}\left(d \mu_{p}\right)=\alpha_{n}\left(d \mu_{p+1}\right) & n, p=0,1,2, \ldots, \\
\beta_{n+1}\left(d \mu_{p}\right)=\beta_{n}\left(d \mu_{p+1}\right) & n=1,2,3, \ldots, p=0,1,2, \ldots
\end{array}
$$

We will now proceed to prove Eq. (89) by construction: by evaluating Eq. (93), for $(n, p)$ at $(s-1, m),(s-2, m+1),(s-3, m+2), \ldots,(0, m+s-1)$ for any $m \geq 0, s \geq 1$ we have the following sequence of equations

$$
\begin{aligned}
\alpha_{s}\left(d \mu_{m}\right) & =\alpha_{s-1}\left(d \mu_{m+1}\right) \\
\alpha_{s-1}\left(d \mu_{m+1}\right) & =\alpha_{s-2}\left(d \mu_{m+2}\right) \\
\alpha_{s-2}\left(d \mu_{m+2}\right) & =\alpha_{s-3}\left(d \mu_{m+3}\right) \\
& \vdots \\
\alpha_{1}\left(d \mu_{m+s-1}\right) & =\alpha_{0}\left(d \mu_{m+s}\right) .
\end{aligned}
$$


Thus by repeated substitution we arrive at

$$
\alpha_{s}\left(d \mu_{m}\right)=\alpha_{0}\left(d \mu_{m+s}\right), \quad m=0,1,2, \ldots, s=1,2,3, \ldots .
$$

We note that this equation is also valid for $s=0$. If we make the change of variable $s=p+n$, $m=0$ in Eq. (99) followed by relabeling indices, we find

$$
\alpha_{m+s}\left(d \mu_{0}\right)=\alpha_{0}\left(d \mu_{m+s}\right) \quad m, s=0,1,2, \ldots
$$

Hence from Eq. (99) and (100), we arrive at (89). Similarly, we prove Eq. (90).

Definition 13 We call the sequence of gapless measures $d \nu_{0}(x), d \nu_{1}(x), d \nu_{2}(x), d \nu_{3}(x)$, ... beta normalised measures, where $d \nu_{0}(x)$ defines the sequence

$$
\bar{\nu}_{n}(x)=\frac{\bar{\nu}_{0}(x)}{\left(P_{n-1}\left(d \nu_{0} ; x\right) \frac{\varphi\left(d \nu_{0} ; x\right)}{2}-Q_{n-1}\left(d \nu_{0} ; x\right)\right)^{2}+\pi^{2} \bar{\nu}_{0}^{2}(x) P_{n-1}^{2}\left(d \nu_{0} ; x\right)}
$$

$n=1,2,3, \ldots$, and $d \nu_{n}(x)=\bar{\nu}_{n}(x) d x \quad n=0,1,2, \ldots$.

Lemma 9 For every sequence of beta normalised measures $\left\{d \nu_{n}\right\}_{n=0}^{n=\infty}$, there always exists a sequence of normalised secondary measures $\left\{d \mu_{n}\right\}_{n=0}^{n=\infty}$ such that

$$
\bar{\nu}_{n}(x)=\beta_{n}\left(d \nu_{0}\right) \bar{\mu}_{n}(x) \quad n=0,1,2, \ldots .
$$

Proof For $n=0$ in Eq. (102) and taking into account corollary (1), we have

$$
\bar{\nu}_{0}(x)=C_{0}\left(d \nu_{0}\right) \bar{\mu}_{0}(x) .
$$

The only additional constraint on $d \mu_{0}$ as compared with any measure $d \mu$, is that $C_{0}\left(d \mu_{0}\right)=$ 1. By calculating the zeroth moment of both sides, we see that relation Eq. (103) satisfies this additional constraint. Now we will proceed by finding an expression for the sequence of normalised measures generated from $d \mu_{0}$ in terms of $d \nu_{0}$. For this we need to find how the quantities $P_{n}\left(d \mu_{0} ; x\right), Q_{n}\left(d \mu_{0} ; x\right), \beta_{n+1}\left(d \mu_{0}\right), n=0,1,2, \ldots$ and $\varphi\left(d \mu_{0} ; x\right)$ can be written in terms of $P_{n}\left(d \nu_{0} ; x\right), Q_{n}\left(d \nu_{0} ; x\right), \beta_{n+1}\left(d \nu_{0}\right), n=0,1,2, \ldots$ and $\varphi\left(d \nu_{0} ; x\right)$ respectively. Using relation Eq. (103) and definition (3), we find

$$
P_{n}\left(d \mu_{0} ; x\right)=\sqrt{C_{0}\left(d \nu_{0}\right)} P_{n}\left(d \nu_{0} ; x\right) \quad n=0,1,2, \ldots .
$$

Using this relation, and definitions (6) and (11), we find

$$
\begin{aligned}
Q_{n}\left(d \mu_{0} ; x\right) & =\frac{Q_{n}\left(d \nu_{0} ; x\right)}{\sqrt{C_{0}\left(d \nu_{0}\right)}} \quad n=0,1,2, \ldots, \\
\varphi\left(d \mu_{0} ; x\right) & =\frac{\varphi\left(d \nu_{0} ; x\right)}{C_{0}\left(d \nu_{0}\right)} .
\end{aligned}
$$

Lemma (7) tells us

$$
\beta_{n}\left(d \mu_{0}\right)=\beta_{n}\left(d \nu_{0}\right) \quad n=1,2,3, \ldots .
$$

Hence using relation Eq. (18), we find

$$
t_{n-1}^{2}\left(d \mu_{0}\right)=t_{n-1}^{2}\left(d \nu_{0}\right)=\beta_{n}\left(d \nu_{0}\right) \quad n=1,2,3, \ldots .
$$


Now substituting Eq. (104), (105), (106), and (108) into Eq. (66),

$$
\bar{\mu}_{n}(x)=\frac{1}{\beta_{n}\left(d \nu_{0}\right)} \frac{\bar{\nu}_{0}(x)}{\left(P_{n-1}\left(d \nu_{0} ; x\right) \frac{\varphi\left(d \nu_{0} ; x\right)}{2}-Q_{n-1}\left(d \nu_{0} ; x\right)\right)^{2}+\pi^{2} \bar{\nu}_{0}^{2}(x) P_{n-1}^{2}\left(d \nu_{0} ; x\right)}
$$

$n=1,2,3, \ldots$. Hence by observing definition (13), we find Eq. (102) for $n=1,2,3, \ldots$.

Theorem $14 A$ sequence of beta normalised measures $d \nu_{0}(x), d \nu_{1}(x), d \nu_{2}(x), \ldots$, satisfy

$$
\begin{array}{ll}
\alpha_{m+n}\left(d \nu_{0}\right)=\alpha_{n}\left(d \nu_{m}\right) & n, m=0,1,2, \ldots, \\
\beta_{m+n}\left(d \nu_{0}\right)=\beta_{n}\left(d \nu_{m}\right) & n, m=0,1,2, \ldots
\end{array}
$$

Proof From lemma (9) we see that $d \mu_{n}$ and $d \nu_{n}$ are related by a constant, hence using lemmas (7) and (8) we find

$$
\begin{array}{ll}
\alpha_{m+n}\left(d \nu_{0}\right)=\alpha_{n}\left(d \nu_{m}\right) & n, m=0,1,2, \ldots, \\
\beta_{m+n}\left(d \nu_{0}\right)=\beta_{n}\left(d \nu_{m}\right) & n=1,2,3, \ldots, m=0,1,2, \ldots
\end{array}
$$

For $\beta_{0}\left(d \nu_{m}\right)$ we find

$$
\beta_{0}\left(d \nu_{m}\right)=\beta_{0}\left(\beta_{m}\left(d \nu_{0}\right) d \mu_{m}\right)=\beta_{m}\left(d \nu_{0}\right) C_{0}\left(d \mu_{m}\right) \quad m=0,1,2, \ldots,
$$

where we have used Eq. (102) followed by Eq. (76) and then corollary (10). But $C_{0}\left(d \mu_{m}\right)=$ $1 m=0,1,2, \ldots$, by definition.

Remark 2 As we will see in section 4 , for a wide range of $d \nu_{0} ; \alpha_{m+n}\left(d \nu_{0}\right), \beta_{m+n}\left(d \nu_{0}\right)$ and $d \nu_{m}$ can be determined analytically. Hence Eq. (110) and Eq. (111) can also be used to find analytical solutions to a wide range of integrals.

Definition 14 We will call the infinite tridiagonal matrix of a measure $d \mu(x)$

$$
\mathcal{J}(d \mu)=\left[\begin{array}{ccccc}
\alpha_{0}(d \mu) & \sqrt{\beta_{1}(d \mu)} & & & \\
\sqrt{\beta_{1}(d \mu)} & \alpha_{1}(d \mu) & \sqrt{\beta_{2}(d \mu)} & & \\
& \sqrt{\beta_{2}(d \mu)} & \alpha_{2}(d \mu) & \sqrt{\beta_{3}(d \mu)} & \\
& & \ddots & \ddots & \ddots
\end{array}\right]
$$

the Jacobi matrix. See [27] for more details.

Definition 15 We will call the matrix

$$
\mathcal{J}_{n}(d \mu)=\left[\begin{array}{ccccc}
\alpha_{n}(d \mu) & \sqrt{\beta_{n+1}(d \mu)} & & & \\
\sqrt{\beta_{n+1}(d \mu)} & \alpha_{n+1}(d \mu) & \sqrt{\beta_{n+2}(d \mu)} & & \\
& \sqrt{\beta_{n+2}(d \mu)} & \alpha_{n+2}(d \mu) & \sqrt{\beta_{n+3}(d \mu)} & \\
& & \ddots & \ddots & \ddots
\end{array}\right]
$$

$n=1,2,3, \ldots$, the $n$th associated Jacobi matrix of the Jacobi matrix $\mathcal{J}(d \mu)$. 
We are now ready to state our second main theorem:

Theorem 15 For Jacobi matrices for which its corresponding measure defines a sequence of normalised secondary measures, there exist an infinite sequence of associated Jacobi matrices corresponding to the sequence of normalised secondary measures. These matrices are formed by crossing-out the first row and column of the previous Jacobi matrix in the sequence:

$$
\mathcal{J}_{n}\left(d \mu_{0}\right)=\mathcal{J}\left(d \mu_{n}\right) \quad n=1,2,3, \ldots
$$

Proof By equating the matrix elements in Eq. (117), we find Eq. (89) and (90). Hence lemma (8) implies Eq. (117).

Corollary 3 Theorem (15) is also valid for any sequence of measures which are proportional to a sequence of normalised secondary measures such as the beta normalised measures.

Proof Given that the Jacobi matrix does not contain the $\beta_{0}$ coefficient, the result follows easily from lemma (7).

\section{Chain mappings of open quantum systems and Markovian embeddings}

\subsection{Chain mappings}

An open quantum system can be represented by a system plus bath (also known as environment) model introduced by Caldeira and Leggett 33 . The Hilbert space of the Hamiltonian for this model $\mathcal{H}$, is the tensor product of the space of the quantum system wavefunctions and the Fock space for the bosonic bath. Formally, $\mathcal{H}=\mathcal{S} \otimes \Gamma(\mathfrak{h})$, where $\mathcal{S}$ is a separable Hilbert space describing the quantum system and $\Gamma(\mathfrak{h})$ is the bosonic Fock space 2 over the one particle space $\mathfrak{h}=L^{2}(\mathbb{R}, d k)$, where $k$ is the boson momentum. This describes a field of scalar bosons. An element $\Psi$ of $\mathcal{H}$, is a sequence $\left\{\Psi^{(n)}\right\}$ with $\Psi^{(n)}=\varphi \otimes \psi^{(n)}$, where $\psi=\left\{\psi^{(n)}\right\} \in \Gamma(\mathfrak{h}), \psi^{(n)}$ is on $\mathbb{R}^{n}$ and the domain of $\varphi \in \mathcal{S}$ is to be specified with the details of the quantum system (we will give some examples later). The elements of $\mathcal{H}$ satisfy $\|\Psi\|:=\|\varphi\|_{\mathcal{S}}\|\psi\|_{\Gamma(\mathfrak{h})}<\infty$, where

$$
\|\Psi\|^{2}=\|\varphi\|_{\mathcal{S}}^{2}\left(\left|\psi^{(0)}\right|^{2}+\sum_{n=1}^{\infty} \int_{k_{\min }}^{k_{\max }} \ldots \int_{k_{\min }}^{k_{\max }} d k_{1} \ldots d k_{n}\left|\psi^{(n)}\left(k_{1}, \ldots, k_{n}\right)\right|^{2}\right),
$$

where each $\psi^{(n)}$ is symmetric in $k_{1}, \ldots, k_{n}$ and $-\infty \leq k_{\min }<k_{\max } \leq \infty$. The Hamiltonian is

$$
H=H_{\mathcal{S}} \otimes \mathbb{I}_{\Gamma(\mathfrak{h})}+\mathbb{I}_{\mathcal{S}} \otimes \int_{k_{\min }}^{k_{\max }} d k g(k) a_{k}^{*} a_{k}+A \otimes \int_{k_{\min }}^{k_{\max }} d k h(k)\left(a_{k}^{*}+a_{k}\right),
$$

$H_{S}$ is a bounded below self-adjoint operator on $\mathcal{S}$ and describes the system dynamics, $H_{E}:=\int_{k_{\min }}^{k_{\max }} d x g(k) a_{k}^{*} a_{k}$ is the Hamiltonian of the environment where $g \geq 0$. This is also

\footnotetext{
2 also known as symmetric Fock space
} 
known in the literature as $d \Gamma(g)$, the second quantisation of $g . a_{k}^{*}, a_{k}$ are creation and annihilation operators with cummutator $\left[a_{k}, a_{k^{\prime}}^{*}\right]=\delta\left(k-k^{\prime}\right)$. They act on each $\psi^{(n)}$ by

$$
\begin{aligned}
\left(a_{k} \psi\right)^{(n)}\left(k_{1}, \ldots, k_{n}\right) & =(n+1)^{1 / 2} \psi^{(n+1)}\left(k, k_{1}, \ldots, k_{n}\right) \quad n=0,1,2, \ldots, \\
\left(a_{k}^{*} \psi\right)^{(n)}\left(k_{1}, \ldots, k_{n}\right) & =n^{-1 / 2} \sum_{j=1}^{n} \delta\left(k-k_{j}\right) \psi^{(n-1)}\left(k_{1}, \ldots, \hat{k}_{j}, \ldots, k_{n}\right) \quad n=1,2,3, \ldots, \\
\left(a_{k}^{*} \psi\right)^{(0)} & =0,
\end{aligned}
$$

where $\hat{k}_{j}$ indicates that $k_{j}$ is omitted. Hence we have that $\left(\mathbb{I}_{\mathcal{S}} \otimes H_{E} \Psi\right)^{(n)}=\sum_{j=1}^{n} g\left(k_{j}\right) \Psi^{(n)}$ on domain $\mathcal{D}\left(\mathbb{I}_{\mathcal{S}} \otimes H_{E}\right)$ of all $\Psi \in \mathcal{H}$ such that $\left\{\left(\mathbb{I}_{\mathcal{S}} \otimes H_{E} \Psi\right)^{(n)}\right\}$ is again in $\mathcal{H}$. Let $\mathfrak{n}$ be the number of bosons operator defined by

$$
(\mathfrak{n} \psi)^{(n)}=n \psi^{(n)}, \quad n=0,1,2, \ldots
$$

on the domain $\mathcal{D}(\mathfrak{n})$ of all $\psi$ in $\Gamma(\mathfrak{h})$ such that $\left\{n \psi^{(n)}\right\}$ is again in $\Gamma(\mathfrak{h})$. $H_{\text {int }}:=A \otimes$ $\int_{k_{\min }}^{k_{\max }} d k h(k)\left(a_{k}^{*}+a_{k}\right)$ describes the interaction between the quantum system and the bosonic environment. $h(k) \in L^{2}\left(\left[k_{\min }, k_{\max }\right]\right)$ and $\int_{k_{\min }}^{k_{\max }} d k h(k)\left(a_{k}^{*}+a_{k}\right)=\int_{k_{\min }}^{k_{\max }} d k h(k) a_{k}^{*}+$ $\int_{k_{\min }}^{k_{\max }} d k h(k) a_{k}$, where

$$
\begin{gathered}
\left(\int_{k_{\min }}^{k_{\max }} d k h(k) a_{k} \psi\right)^{(n)}\left(k_{1}, \ldots, k_{n}\right)=(n+1)^{1 / 2} \int_{k_{\min }}^{k_{\max }} h(k) \psi^{(n+1)}\left(k, k_{1}, \ldots, k_{n}\right) d k \quad n=0,1,2, \ldots \\
\left(\int_{k_{\min }}^{k_{\max }} d k h(k) a_{k}^{*} \psi\right)^{(n)}\left(k_{1}, \ldots, k_{n}\right)=n^{-1 / 2} \sum_{j=1}^{n} h\left(k_{j}\right) \psi^{(n-1)}\left(k_{1}, \ldots, \hat{k}_{j}, \ldots, k_{n}\right) \quad n=1,2,3, \ldots,(124) \\
\left(\int_{k_{\min }}^{k_{\max }} d k h(k) a_{k}^{*} \psi\right)^{(0)}
\end{gathered}
$$

is a well defined self-conjugate operator (also known in the literature as $\Phi_{s}(h \sqrt{2}$ ), the $S e g a l$ field operator) on $\mathcal{D}\left(\mathfrak{n}^{1 / 2}\right)$. $A$ is any bounded below self-adjoint operator with domain in $\mathcal{S}$ such that $H$ is a well defined Hamiltonian with domain in $\mathcal{H}$. See Theorem (16) and remark (3) for more details. We call $H_{0}=H_{\mathcal{S}} \otimes \mathbb{I}_{\Gamma(\mathfrak{h})}+\mathbb{I}_{\mathcal{S}} \otimes H_{E}$ the free Hamiltonian and define $\omega_{\text {max }}:=\sup g, \omega_{\text {min }}:=\inf g . \omega_{\text {min }}$ is sometimes called as the mass of the bosons. The case of massless bosons, i.e. when $\omega_{\min }=0$, is of particular interest.

Theorem 16 If $A$ is a bounded operator 3 on $S, H$ is self-adjoint on $\mathcal{D}(H)=\mathcal{D}\left(H_{0}\right)$ if

$$
\int_{k_{\min }}^{k_{\max }} \frac{h^{2}(k)}{g(k)} d k<\infty
$$

Proof This is a well-known result from the literature. See e.g. 34], section 7.

\footnotetext{
3 i.e. $\|A \varphi\|_{\mathcal{S}} \leq C\|\varphi\|_{\mathcal{S}} \forall \varphi \in \mathcal{D}(A), 0 \leq C<\infty$.
} 
Remark 3 In the case that $A$ is an unbounded operator on $\mathcal{S}$, there also exist conditions under which $H$ can be shown to be self-adjoint on some appropriate domain. See Theorem 2.2 of 35 .

Two examples of Hamiltonians satisfying Lemma (16) are the Spin-Boson model, where $H_{\mathcal{S}}=\alpha \sigma_{z}, A=\sigma_{x}$, where $\alpha$ is a positive constant and $\sigma_{x}, \sigma_{z}$ are the Pauli matrices. $\varphi=\left(\gamma_{1}, \gamma_{2}\right)^{T}$ with $\gamma_{1}, \gamma_{2} \in \mathbb{C}$. Another example is when the quantum system is comprised of $N$ spinless nucleons of mass $M>0$. In this case $H_{\mathcal{S}}=-\sum_{j=1}^{N} \nabla_{j}^{2} /(2 M)$ where $\nabla_{j}^{2}$ is the Laplacian in the variable $x_{j}$ with usual domain which makes it a self-adjoint operator and $\varphi=\varphi\left(x_{1}, \ldots, x_{N}\right)$. A, for example could be the operator of multiplication by a positive function $f \in L^{2}\left(\mathbb{R}^{N}\right)$.

Additional assumptions: In order to prove the results of this paper, we will need to make some assumptions in addition to those which make the Hamiltonian (119) well-defined:

A1. $g(x)$ is invertable and differentiable on the interval $\left[k_{\min }, k_{\max }\right]$ satisfying $d g(x) / d x \geq 0$ or $d g(x) / d x \leq 0$. 4

A2. $J(x)$ has finite moments on the interval $\left[\omega_{\min }, \omega_{\max }\right] 5$

Corollary 4 If $A$ is a bounded operator on $\mathcal{S}$, and assumptions A1. and A2. are satisfied, then $H$ is self-adjoint on $\mathcal{D}(H)=\mathcal{D}\left(H_{0}\right)$.

Proof We can use $A 1$ to make the change of variable $k=g^{-1}(x)$ in Eq. (126) and hence write the integrand in terms on J. Now $A 2$. implies that Theorem (16) is satisfied.

Physically $g(x)$ represents the dispersion relation of the environment and $h(x)$ determines the system-environment coupling strength. Together they determine the spectral density in the following way

Definition 16 We call the function $J(x)$ the spectral density,

$$
J(\omega)=\pi h^{2}\left(g^{-1}(\omega)\right)\left|\frac{d g^{-1}(\omega)}{d \omega}\right|
$$

where $g^{-1}(g(x))=g\left(g^{-1}(x)\right)=x, \operatorname{Dom}[J] \in\left[\omega_{\text {min }}, \omega_{\text {max }}\right]$, and $|$.$| denotes the absolute$ value. Recall that $\omega_{\max }$ can be finite or infinite such that the interval $\left[\omega_{\min }, \omega_{\max }\right]$ can be bounded or unbounded.

Analogously to the measures (definition (10), we call the spectral density gapless if $J$ has support on all of the interval $\left(\omega_{\min }, \omega_{\max }\right)$ and gapped if the support of $J$ is a disjoint set of intervals.

Remark 4 As we will see later in the proof to theorem (17) (more specifically in Eq. 160), we use $J$ in the definition of a measure with weight function $M^{q}(x)$ in such a way that the measure will be gapped iff $J$ is gapped. Hence if we want to form a gapless measure, in accordance with definition (1), we need to use a gapless spectral density. For cases where this does not hold, i.e. $J$ is gapped, we can define a set of spectral densities corresponding to

\footnotetext{
${ }^{4}$ Note however that if this is not the case, there should be ways to get around this difficulty.

${ }^{5}$ Note that if this is not the case because $\left[\omega_{\min }, \omega_{\max }\right]$ is an unbounded interval, one can define a cut-off hamiltonian such that the interval is bounded and the theorems developed here will then apply.
} 


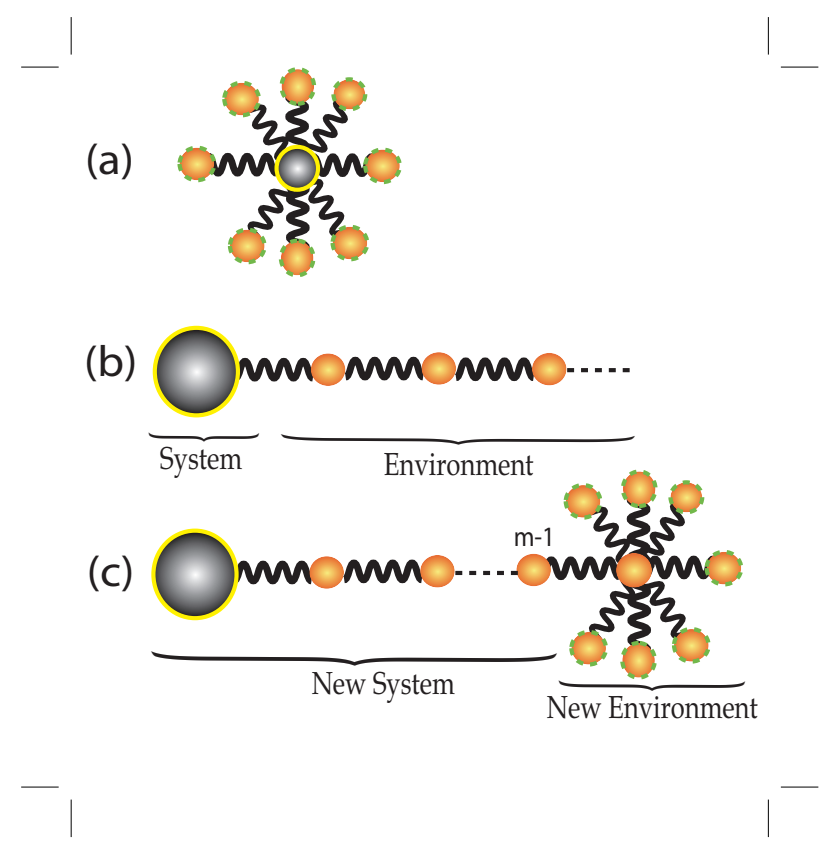

Fig. 1 (a) The initial system-environment Hamiltonian before the mapping has been performed: the system (depicted by the gray ball in the centre) couples directly to the degrees of freedom of the enviroment (orange balls). (b) The system-environment Hamilatonian after the chain mapping has been performed: the environment has been mapped onto a semi-infinite chain of nearest neighbour interactions where the system now only couples to the first element in the chain. (c) The systemenvironment Hamiltonian after the $m$ th environmental degree of freedom has been embedded: the new system, interacts with the new enviroment via the $m$ th residual spectral density $J_{m}(x)$.

gapless measures by redefining their domain such that they contain only non positive values at the boundaries of their domain. It is not necessary to do this, but has some advantages as some additional theorems will then apply. Also see remark (7).

Lemma 10 Given a measure $d \mu(x)$ with $I=\left[G_{q}\left(g\left(k_{\min }\right)\right), G_{q}\left(g\left(k_{\max }\right)\right)\right]$ if $g$ is nondecreasing and $I=\left[G_{q}\left(g\left(k_{\max }\right)\right), G_{q}\left(g\left(k_{\min }\right)\right)\right]$ if $g$ is non-increasing and its corresponding monic orthogonal polynomials $\left\{\pi_{n}(d \mu ; x)\right\}_{n=0}^{n=\infty}$, the following holds. One can construct the set of functions $\left\{\underline{\pi}_{n}(d \mu ; x)\right\}_{n=0}^{n=\infty}$,

$$
\underline{\pi}_{n}(d \underline{\mu} ; x):=\pi_{n}\left(d \mu ; G_{q}(g(x))\right), \quad n=0,1,2, \ldots,
$$

where

$$
G_{q}(x):=\frac{-q\left(1+q^{2}\right)+2 \sqrt{q^{4}+4\left(1-q^{2}\right) x^{2}}}{4\left(1-q^{2}\right)} \quad x \geq 0, q \in[0,1]
$$

which satisfy the 3 -term recurrence relation

$$
\begin{gathered}
\underline{\pi}_{n+1}(\underline{d \mu} ; x)=\left(G_{q}(g(x))-\alpha_{n}(d \mu)\right) \underline{\pi}_{n}(\underline{d \mu} ; x)-\beta_{n}(d \mu) \underline{\pi}_{n-1}(\underline{d \mu} ; x), \\
\underline{\pi}-1 \underline{(d \mu} ; x):=0 \quad n=0,1,2, \ldots
\end{gathered}
$$


and are orthogonal with respect to the measure $d \underline{\mu}(x)$

$$
\int_{k_{\min }}^{k_{\max }} \underline{\pi}_{n}(\underline{d \mu} ; x) \underline{\pi}_{m}(\underline{d \mu} ; x) \underline{\mu}(x) d x=\left\langle\pi_{n}(d \mu), \pi_{n}(d \mu)\right\rangle_{\bar{\mu}} \delta_{n m} \quad n, m=0,1,2, \ldots,
$$

where $\underline{d \mu}(x)=: \underline{\bar{\mu}}(x) d x$, with

$$
\underline{\bar{\mu}}(x)=\bar{\mu}\left(G_{q}(g(x))\right)\left|\frac{d G_{q}(g(x))}{d x}\right|,
$$

with $I=\left[k_{\min }, k_{\max }\right]$. Furthermore, the converse is true: given the set of functions $\left\{\underline{\pi}_{n}(d \mu ; x)\right\}_{n=0}^{\infty}$ defined in terms of a set of monic polynomials $\left\{\pi_{n}(x)\right\}_{n=0}^{\infty}$ which satisfy Eqs. (130) and (132) for a weight function $\bar{\mu}$ defined in terms of a weight function $\bar{\mu}$ with $I=\left[G_{q}\left(g\left(k_{\min }\right)\right), G_{q}\left(g\left(k_{\max }\right)\right)\right]$ if $g$ is non-decreasing and $\bar{I}=\left[G_{q}\left(g\left(k_{\max }\right)\right), G_{q}\left(g\left(k_{\min }\right)\right)\right]$ if $g$ is non-increasing, then the set $\left\{\pi_{n}(x)\right\}_{n=0}^{\infty}$ are the corresponding monic orthogonal polynomials for the weight function $\bar{\mu}$.

Proof Eqs. (132) and (133) follow from perfoming a change of variable in $\left\langle\pi_{n}(d \mu), \pi_{m}(d \mu)\right\rangle_{\bar{\mu}}=\left\langle\pi_{n}(d \mu), \pi_{n}(d \mu)\right\rangle_{\bar{\mu}} \delta_{n m}$ and noting that $d G_{q}(g(x)) / d x \geq 0$ if $g$ is nondecreasing and $d G_{q}(g(x)) / d x \leq 0$ if $g$ is non-increasing. Eq. (130) follows from a change of variable in Eq. (77). The fact that the converse is true follows from the fact that $G_{q}$ and $g$ have well-defined inverse functions.

Theorem 17 There is a class of quantum systems linearly coupled with a reservoir with spectral density $J(x)$ which are equivalent to semi-infinite chains with only nearest-neighbors interactions, where the system only couples to the first site in the chain. More specifically, starting from an initial Hamiltonian,

$$
H=H_{S} \otimes \mathbb{I}_{\Gamma(\mathfrak{h})}+\mathbb{I}_{\mathcal{S}} \otimes \int_{k_{\min }}^{k_{\max }} d k g(k) a_{k}^{*} a_{k}+A \otimes \int_{k_{\min }}^{k_{\max }} d k h(k)\left(a_{k}^{*}+a_{k}\right),
$$

satisfying the assuptions $A 1, A 2$, there exists, for every $q \in[0,1]$, a countably infinite set of new creation $b_{n}^{*}(q)$ and annihilation $b_{n}(q)$ operators

$$
\begin{aligned}
& b_{n}^{*}(q)=\int_{k_{\min }}^{k_{\max }} d x U_{n}\left(d \lambda^{q} ; x\right)\left[\cosh r_{q}(g(x)) a_{x}^{*}-\sinh r_{q}(g(x)) a_{x}\right] \quad n=0,1,2, \ldots \\
& b_{n}(q)=\int_{k_{\min }}^{k_{\max }} d x U_{n}\left(d \lambda^{q} ; x\right)\left[\cosh r_{q}(g(x)) a_{x}-\sinh r_{q}(g(x)) a_{x}^{*}\right]
\end{aligned}
$$

well-defined on $\mathcal{D}\left(\mathfrak{n}^{1 / 2}\right)$ which satisfy the commutation relations

$$
\left[b_{n}(q), b_{m}^{*}(q)\right]=\delta_{n m} \quad n, m=0,1,2, \ldots,
$$

with transformed Hamiltonian

$$
\begin{aligned}
: H:= & H_{S} \otimes \mathbb{I}_{\Gamma(\mathfrak{h})}+\mathbb{I}_{\mathcal{S}} \otimes H_{E, q}+H_{\text {int }, q} \\
H_{E, q}= & \sum_{n=0}^{\infty}\left\{E_{1 n}(q)\left(b_{n}^{*}(q) b_{n}^{*}(q)+b_{n}(q) b_{n}(q)\right)+E_{2 n}(q) b_{n}^{*}(q) b_{n}(q)\right. \\
& +E_{3 n}(q)\left(b_{n}^{*}(q) b_{n+1}^{*}(q)+b_{n}(q) b_{n+1}(q)\right) \\
& \left.+E_{4 n}(q)\left(b_{n}^{*}(q) b_{n+1}(q)+b_{n}(q) b_{n+1}^{*}(q)\right)\right\} \\
H_{\text {int }, q}= & E_{5}(q) A \otimes\left(b_{0}^{*}(q)+b_{0}(q)\right) .
\end{aligned}
$$


where $E_{1 n}(q), E_{2 n}(q), E_{3 n}(q), E_{4 n}(q), E_{5}(q) \in \mathbb{R}$ and $q \in[0,1]$ is a free parameter of the mapping which determines the particular version. $H:$ is to indicate that the hamiltonian $H$ has been renormalized 6 . The constants $E_{1 n}(q), E_{2 n}(q), E_{3 n}(q), E_{4 n}(q), E_{5}(q)$ are determined when a particular spectral density $J(x)$ and value of $q$ are specified. Expressions for the functions $U\left(d \lambda^{q} ; x\right)$ and $r_{q}(x)$ are derived in the proof. For a pictorial representation of this theorem, see (a) and (b) of figure (1).

Proof The proof is by construction. Let us start by defining a local transformation of the creation and annihilation operators $a_{x}^{*}$ and $a_{x}$ into another set, $c_{x}$ and $c_{x}^{*}$, which preserves the commutation relations. We can do this via a so called Bogoliubov transformation 38 ]

$$
\begin{aligned}
& a_{x}=\cosh \left(r_{q}(g(x))\right) c_{x}+\sinh \left(r_{q}(g(x))\right) c_{x}^{*}, \\
& a_{x}^{*}=\cosh \left(r_{q}(g(x))\right) c_{x}^{*}+\sinh \left(r_{q}(g(x))\right) c_{x},
\end{aligned}
$$

where $r_{q}(x) \in \mathbb{R}$ and $\left[a_{x}, a_{y}^{*}\right]=\left[c_{x}, c_{y}^{*}\right]=\delta(x-y)$. Notice how we have parametrised the argument of the cosh and sinh functions in terms of $r_{q}(g(x))$. As we will see later, there are a familiy of functions $r_{q}(x)$ for which it is usefull to perform this tranformation. The $q$ is to denote which particular function is being used and will become clear soon. 7 If we now parameterise $r_{q}(x)$ by introducing a new function $\xi_{q}(x)$ through $r_{q}(x)=\ln \xi_{q}(x)$ where $\xi_{q}(x) \in[0,+\infty)$ using Eq. (143) and (144) we find after renormalising

$$
\begin{aligned}
: \int_{k_{\min }}^{k_{\max }} d x g(x) a_{x}^{*} a_{x}:= & \int_{k_{\min }}^{k_{\max }} d x \frac{g(x)}{4 \xi_{q}^{2}(g(x))}\left(\left(\xi_{q}^{4}(g(x))-1\right)\left(c_{x}^{*} c_{x}^{*}+c_{x} c_{x}\right)\right. \\
& \left.+2\left(\xi_{q}^{4}(g(x))+1\right) c_{x}^{*} c_{x}\right) .
\end{aligned}
$$

System-environment term simplifies to

$$
A \otimes \int_{k_{\min }}^{k_{\max }} d x h(x)\left(a_{x}+a_{x}^{*}\right)=A \otimes \int_{k_{\min }}^{k_{\max }} d x h(x) \xi_{q}(g(x))\left(c_{x}^{*}+c_{x}\right) .
$$

For appropriate choice of the function $\xi_{q}(x)$, we can define a measure

$$
\begin{aligned}
& d \underline{\lambda}^{q}(x)=\underline{M}^{q}(x) d x, \\
& \underline{M}^{q}(x)=h^{2}(x) \xi_{q}^{2}(g(x)) .
\end{aligned}
$$

With the set of monic polynomials $\left\{\pi_{n}\left(d \lambda^{q} ; x\right)\right\}_{n=0}^{\infty}$ with measure $d \lambda^{q}(x)$, we are able to construct the set $\left\{\underline{\pi}_{n}\left(d \underline{\lambda}^{q} ; x\right)\right\}_{n=0}^{\infty}$ of orthogonal functions with respect to measure $d \underline{\lambda}^{q}(x)$ defined in Lemma [10. We can use these to define the set of functions $\left\{U_{n}\left(d \lambda^{q} ; x\right)\right\}_{n=0}^{\infty}$ through the relation

$$
U_{n}\left(d \lambda^{q} ; x\right)=\frac{\underline{\pi}_{n}\left(d \underline{\lambda}^{q} ; x\right) \sqrt{\underline{M}^{q}(x)}}{\sqrt{\left\langle\underline{\pi}_{n}\left(d \underline{\lambda}^{q}\right), \underline{\pi}_{n}\left(d \underline{\lambda}^{q}\right) \underline{M}^{q}\right.}}=\frac{\underline{\pi}_{n}\left(d \underline{\lambda}^{q} ; x\right) h(x) \xi_{q}(g(x))}{\sqrt{\left\langle\underline{\pi}_{n}\left(d \underline{\lambda}^{q}\right), \underline{\pi}_{n}\left(d \underline{\lambda}^{q}\right){\underline{M^{q}}}^{q}\right.}} .
$$

\footnotetext{
${ }^{6}$ Specifically, a constant factor $C \mathbb{I}_{\mathcal{S}} \otimes \mathbb{I}_{\Gamma}(\mathfrak{h}), C \in \mathbb{R}$ has been neglected.

7 We can replace the $\cosh$ and sinh functions with $\cos$ and $\sin$ functions for fermions. By doing so, we could work out a different version of this theorem which would be valid for the case where the initial bosonic creation-anhilation operators $\left\{a_{x}, a_{x}^{*}\right\} x \in\left[k_{\min }, k_{\max }\right]$ where fermionic instead
} 
We can now define the set of creation and annihilation operators of the chain (we will specify their domain and show that they are well-defined later in the proof)

$$
\begin{aligned}
& b_{n}^{*}(q)=\int_{k_{\min }}^{k_{\max }} d x U_{n}\left(d \lambda^{q} ; x\right) c_{x}^{*}(q), \\
& b_{n}(q)=\int_{k_{\min }}^{k_{\max }} d x U_{n}\left(d \lambda^{q} ; x\right) c_{x}(q) .
\end{aligned}
$$

Substituting the inverse relations

$$
\begin{aligned}
& c_{x}^{*}(q)=\sum_{n=0}^{\infty} U_{n}\left(d \lambda^{q} ; x\right) b_{n}^{*}(q), \\
& c_{x}(q)=\sum_{n=0}^{\infty} U_{n}\left(d \lambda^{q} ; x\right) b_{n}(q),
\end{aligned}
$$

into Eq. (146) we obtain

$$
A \otimes \int_{k_{\min }}^{k_{\max }} d x h(x)\left(a_{x}+a_{x}^{*}\right)=\sqrt{\beta_{0}\left(d \lambda^{q}\right)} A \otimes\left(b_{0}^{*}(q)+b_{0}(q)\right) .
$$

So we note that for all functions $\xi_{q}(x)$ that result in a valid measure Eq. (147), one can achieve a coupling between system and reservoir which only interacts with the first element in the chain.

Now we will examine carefully what type of chain can be generated via this transformation.

Using the orthogonality conditions of the orthogonal polynomials and Eq. (152) and (153), we can transform terms of the form $\int_{k_{\min }}^{k_{\max }} c_{a x} c_{b x} d x$ into terms of the form $\sum_{n=0}^{\infty} W_{n} b_{a n} b_{b n}$ where the $W_{n}$ 's are constants and the sub indices $a, b=0$ denote that the operator is an annihilation operator and $a, b=1$ denote that they are creation operators. Also, we can transform terms of the form $\int_{k_{\min }}^{k_{\max }} G_{q}(g(x)) c_{a x} c_{b x} d x$ into $\sum_{n=0}^{\infty} W_{n} b_{a n} b_{b n}+W_{1} b_{a(n+1)} b_{b n}+$ $W_{2 n} b_{a n} b_{b(n+1)}$ by using the three term recurrence relations Eq. (130) to eliminate the $G_{q}(g(x))$. We can also map terms of the form $\int_{k_{\min }}^{k_{\max }}\left(G_{q}(g(x))\right)^{k} c_{a x} c_{b x} d x \quad k=2,3,4 \ldots$ using the three term recurrence relations in Eq. (130) $k$ times, but this would result in every chain site coupling to its $k$ th nearest neighbours. Given this resoning, we propose a trial solution to Eq. (145) which will reduce it to a chain of nearest neighbour interactions. This is:

$$
\begin{aligned}
& \frac{g(x)}{4 \xi_{q}^{2}(g(x))}\left(\xi_{q}^{4}(g(x))-1\right)=c_{1}+g_{1} G_{q}(g(x)), \\
& \frac{g(x)}{2 \xi_{q}^{2}(g(x))}\left(\xi_{q}^{4}(g(x))+1\right)=c_{2}+g_{2} G_{q}(g(x)) .
\end{aligned}
$$

Not all values of the real constants $c_{1}, c_{2}, g_{1}, g_{2}$ will result in a valid trial solution. We will parametrise a valid set of these constants in terms of $q$ and hence there will be a different valid solution for the functions $\xi_{q}$ and $G_{q}$ for different values of $q$. This is where the $q$ dependency enters in the proof. A valid and usefull parametrisation is $2 g_{1}=q, g_{2}=1,8 c_{1}=$ 
$-q^{2}, 4 c_{2}=q, q \in[0,1]$. Solving Eqs. (155) and (156) for $G_{q}$ and $\xi_{q}$ for this parametrisation of the constants gives us

$$
\begin{array}{cc}
G_{q}(x)=\frac{-q\left(1+q^{2}\right)+2 \sqrt{q^{4}+4\left(1-q^{2}\right) x^{2}}}{4\left(1-q^{2}\right)} & x \geq 0, \\
\xi_{q}(x)=\left[\frac{q(1-q)+(1+q) 4 G_{q}(x)}{q(1+q)+(1-q) 4 G_{q}(x)}\right]^{1 / 4} & x \geq 0 .
\end{array}
$$

Now that we have explicit expresions for $G_{q}$ and $\xi_{q}$, we will derive an expression for the measure $d \lambda^{q}(x)=M^{q}(x) d x$ from $d \underline{\lambda}^{q}=\underline{M}^{q}(x) d x$. From Eqs (148), (1477) and (133), we have

$$
h^{2}(x) \xi^{2}(g(x))=\underline{M}^{q}(x)=M^{q}\left(G_{q}(g(x))\right)\left|\frac{d G_{q}(g(x))}{d x}\right| .
$$

Solving this for $M^{q}$ using Eqs (157), (158) and definition (16), we find

$$
M^{q}(x)=\frac{J\left(G_{q}^{-1}(x)\right)}{\pi} \frac{\left(1+q^{2}\right) q+4\left(1-q^{2}\right) x}{(1+q) q+4(1-q) x},
$$

where $G_{q}\left(G_{q}^{-1}(x)\right)=G_{q}^{-1}\left(G_{q}(x)\right)=x$ and is given by

$$
G_{q}^{-1}(x)=\frac{1}{4} \sqrt{[q(1-q)+4(1+q) x][q(1+q)+4(1-q) x]} \quad x \geq \frac{-q(1-q)}{4(1+q)},
$$

and we recall from Lemma 10, that the interval $I$ for $d \lambda^{q}=M^{q}(x)$ is $I=\left[G_{q}\left(g\left(k_{\min }\right)\right), G_{q}\left(g\left(k_{\max }\right)\right)\right]$ if $g$ is a non-decreasing function and $I=\left[G_{q}\left(g\left(k_{\max }\right)\right), G_{q}\left(g\left(k_{\min }\right)\right)\right]$ if $g$ is a non-increasing function. Noting that $M^{q}$ has finite moments on $I$ if $J$ does on $\left[\omega_{\min }, \omega_{\max }\right]$ and taking into account assumption $A 2$. and Eqs. (150), (151), (157), (158), we find that $f_{1, q, n}(x):=$ $U_{n}\left(d \lambda^{q} ; x\right) \cosh r_{q}(g(x)), f_{2, q, n}(x):=U_{n}\left(d \lambda^{q} ; x\right) \sinh r_{q}(g(x)) \in L^{2}\left(\left[k_{\min }, k_{\max }\right]\right) . b_{n}^{*}(q) \Psi^{(n)}$ and $b_{n}(q) \Psi^{(n)}$ are defined as in Eqs (124) to (125) when exchanging $h$ for $f_{1, q, n}$ and $f_{2, q, n}$ accordingly, and hence $b_{n}^{*}(q)$ and $b_{n}(q)$ are well-defined operators on $\mathcal{D}\left(\mathfrak{n}^{1 / 2}\right)$. Furthermore we can verify that they satisfy

$$
\left(\Psi_{1}, b_{n}(q) \Psi_{2}\right)=\left(b_{n}^{*}(q) \Psi_{1}, \Psi_{2}\right),
$$

for all $\Psi_{1}, \Psi_{2} \in \mathcal{D}\left(\mathfrak{n}^{1 / 2}\right)$ hence confirming that $b_{n}^{*}(q)$ is the adjoint of $b_{n}(q)$. The commutation relation $\left[b_{n}(q), b_{m}^{*}(q)\right]=\delta_{n, m}$ follows from the orthogonality conditions for the monic polinomials and the commutator relation $\left[a_{k}, a_{k^{\prime}}^{*}\right]=\delta\left(k-k^{\prime}\right)$. Now let us perform the trasnformation. After substituting Eqs. (152) and (153) into Eq. (145) and using the orthogonality conditions and 3-term recurrence relations of Lemma 10, we find

$$
\begin{aligned}
& : \int_{k_{\min }}^{k_{\max }} d x g(x) a_{x}^{*} a_{x}: \\
= & \sum_{n=0}^{\infty}\left\{\left(\frac{q}{2} \alpha_{n}(q)-\frac{q^{2}}{8}\right)\left(b_{n}^{*}(q) b_{n}^{*}(q)+b_{n}(q) b_{n}(q)\right)+\left(\alpha_{n}(q)+\frac{q}{4}\right) b_{n}^{*}(q) b_{n}(q)\right. \\
+ & \left.\sqrt{\beta_{n+1}(q)}\left(q\left(b_{n}^{*}(q) b_{n+1}^{*}(q)+b_{n}(q) b_{n+1}(q)\right)+\left(b_{n}^{*}(q) b_{n+1}(q)+b_{n}(q) b_{n+1}^{*}(q)\right)\right)\right\},
\end{aligned}
$$

where $\alpha(q):=\alpha\left(d \lambda^{q}\right), \beta(q):=\beta\left(d \lambda^{q}\right)$. 
Remark 5 Note that the chain mapping, theorem (17), the spectral density can represent continuous modes and/or discrete modes. The discrete modes are represented by dirac delta distributions and the continuous modes by continuous functions. In the case of $N$ discrete modes only, the chain mapping will map the system onto a chain with $N$ sites. Mathematically, the reason for this is because the inner product of the measure (2) in this case can only be defined for functions living in the space spanned by a set of $N$ orthogonal polynomials of finite degree (See discrete measure, page 4 following Theorem 1.8 of [27]). Physically, this is because the number of degrees of freedom corresponding to the Hamiltonian after and before the mapping have to be the same. Also see remark (7) regarding other features of spectral densities.

In addition, it is also worth noting that if the system interacts linearly with more than one environment through different system operators $A$ for each environment, then one can easily generalise the results of the generalised mapping (theorem (17)) such that we can map the Hamiltonian onto a Hamiltonian where the system interacts with more than one chain (i.e. one chain for each environment).

Corollary 5 The generalised mapping Eq. (138) reduces to

$H=H_{S} \otimes \mathbb{I}_{\Gamma(\mathfrak{h})}+\sqrt{\beta_{0}(0)} A \otimes\left(b_{0}(0)+b_{0}^{*}(0)\right)+\mathbb{I}_{\mathcal{S}} \otimes \sum_{n=0}^{\infty} \alpha_{n}(0) b_{n}^{*}(0) b_{n}(0)+\sqrt{\beta_{n+1}(0)}\left(b_{n+1}^{*}(0) b_{n}(0)+\right.$ h.c. $)$

when $q=0$. We also find

$$
M^{0}(x)=\frac{J(x)}{\pi}
$$

with

$$
I=\left[\omega_{\min }, \omega_{\max }\right]
$$

and

$$
\begin{aligned}
G_{0}(x) & =x \\
b_{n}^{*}(0) & =\int_{k_{\min }}^{k_{\max }} \frac{d x \pi_{n}\left(d \lambda^{0} ; g(x)\right)}{\sqrt{\left\langle\pi_{n}\left(d \lambda^{0}\right), \pi_{n}\left(d \lambda^{0}\right)\right\rangle_{M^{0}}}} \sqrt{\frac{J(g(x))}{\pi}\left|\frac{d g(x)}{d x}\right|} a_{x}^{*}, \quad n=0,1,2, \ldots
\end{aligned}
$$

If we set $g(x)=\kappa x, \omega_{\text {min }}=0$, for some $\kappa>0$, then this chain representation reduces to the result found in 19, 18. Otherwise, the chain coefficients are the same, but here the correct relation between the operators $\left\{a_{x}, a_{x}^{*}\right\}$ and $\left\{b_{n}(0)\right\}_{n=0}^{\infty}$ is given.

Proof Follows from setting $q=0$ in theorem (17) and simplifying the resultant expressions.

Given that the coupling of the chain elements is excitation number preserving, the elementary excitations of the chain can be viwed as particles hopping on a 1d lattice. We therefore make the following definition.

Definition 17 We shall refer to the transformation described in corollary (5) as the particle mapping. 
Corollary 6 When $q=1$, the generalised mapping Eq. (138) reduces to

$$
: H:=H_{S} \otimes \mathbb{I}_{\Gamma(\mathfrak{h})}+\sqrt{\beta_{0}(1)} A \otimes X_{0}+\mathbb{I}_{\mathcal{S}} \otimes \sum_{n=0}^{\infty}\left(\sqrt{\beta_{n+1}(1)} X_{n} X_{n+1}+\frac{\alpha_{n}(1)}{2} X_{n}^{2}+\frac{1}{2} P_{n}^{2}\right) .
$$

where $X_{n}$ and $P_{n}$ are position and momentum operators, $X_{n}:=\left(b_{n}^{*}(1)+b_{n}(1)\right), P_{n}:=$ $i\left(b_{n}^{*}(1)-b_{n}(1)\right) / 2$, on $\mathcal{D}\left(\mathfrak{n}^{1 / 2}\right), n=0,1,2, \ldots$ We also find

$$
M^{1}(x)=\frac{J(\sqrt{x})}{\pi}
$$

with

$$
I=\left[\omega_{\min }^{2}, \omega_{\max }^{2}\right]
$$

and

$$
\begin{aligned}
G_{1}(x) & =x^{2}, \\
b_{n}^{*}(1) & =\int_{k_{\min }}^{k_{\max }} \frac{d x \pi_{n}\left(d \lambda^{1} ; g^{2}(x)\right)}{\sqrt{\left\langle\pi_{n}\left(d \lambda^{1}\right), \pi_{n}\left(d \lambda^{1}\right)\right\rangle_{M^{1}}}} \sqrt{\frac{J(g(x))}{\pi}\left|\frac{d g(x)}{d x}\right|}\left(\frac{2 g(x)+1}{2} a_{x}^{*}-\frac{2 g(x)-1}{2} a_{x}\right) .
\end{aligned}
$$

Proof Follows from setting $q=1$ in theorem (17) and simplifying the resultant expressions.

Given that the coupling of the chain elements in Eq. (170) resemble that of springs obeying hooks law, the elementary excitations are phonons such as in solid state physics. We therefore make the following definition.

Definition 18 We shall refer to the transformation described in corollary (6) as the phonon mapping.

Remark 6 In light of definitions (17) and (18), we note that Eq. (138) interpolates between the two solutions.

We will now re-write the generalised mapping in terms of Jacobi matrices, this is to illustrate the connection with Jacobi matrix theory and to write the Hamiltonian in a more compact form.

Corollary $\mathbf{7}$ The generalised mapping in terms of Jacobi matrices is:

$$
\begin{aligned}
H & =H_{S} \otimes \mathbb{I}_{\Gamma(\mathfrak{h})}+\sqrt{\beta_{0}(q)} A \otimes\left(b_{0}^{*}+b_{0}\right) \\
& +\mathbb{I}_{\mathcal{S}} \otimes \frac{q}{2}\left[\mathbf{b}_{0}^{T}\left(\mathcal{J}\left(d \lambda^{q}\right)-\frac{q}{4} \mathbb{I}\right) \mathbf{b}_{0}+\text { h.c. }\right]+\mathbb{I}_{\mathcal{S}} \otimes \mathbf{b}_{0}^{*}\left(\mathcal{J}\left(d \lambda^{q}\right)+\frac{q}{4} \mathbb{I}\right) \mathbf{b}_{0},
\end{aligned}
$$

where

$$
\begin{aligned}
& \mathbf{b}_{n}^{*}=\mathbf{b}_{n}^{*}(q):=\left(b_{n}^{*}(q), b_{n+1}^{*}(q), b_{n+2}^{*}(q), b_{n+3}^{*}(q), \ldots\right)^{T} \\
& \mathbf{b}_{n}=\mathbf{b}_{n}(q):=\left(b_{n}(q), b_{n+1}(q), b_{n+2}(q), b_{n+3}(q), \ldots\right)^{T} \quad n=0,1,2, \ldots
\end{aligned}
$$


Let us define (for every $q \in[0,1]$ ) the orthonormal Fock basis

$$
F_{q}:=\left\{\frac{b_{0}^{* m_{0}}(q)|0\rangle}{\sqrt{m_{0} !}} \otimes \frac{b_{1}^{* m_{1}}(q)|0\rangle}{\sqrt{m_{1} !}} \otimes \frac{b_{2}^{* m_{2}}(q)|0\rangle}{\sqrt{m_{2} !}} \otimes \ldots\right\}_{\left\{m_{n}\right\}_{n=0}^{\infty}=0}^{\infty}
$$

where the creation/annihilation operators (which we recall satisfy $\left[b_{n}(q), b_{m}^{*}(q)\right]=\delta_{n, m}$, $\left.\left[b_{n}(q), b_{m}(q)\right]=\left[b_{n}^{*}(q), b_{m}^{*}(q)\right]=0\right)$ act on the kets $|n\rangle$ in the standard way: $b_{m}^{*}(q)|n\rangle=$ $\sqrt{n+1}|n+1\rangle, b_{m}(q)|n\rangle=\sqrt{n}|n-1\rangle$. Let $\mathcal{K}_{n}$ be the space of all complex linear combinations of $\left\{\frac{b_{n}^{* m}(q)|0\rangle}{\sqrt{m !}}\right\}_{m=0}^{\infty}$ such that $\langle\gamma \mid \gamma\rangle<\infty$ for all $|\gamma\rangle \in \mathcal{K}_{n}$.

Definition 19 We call $m$ th embedded system to the new system-environment interaction produced when the new system is composed of the initial system plus the first $m$ sites of the chain formed by the environment in the chain representation, this is to say, the quantum system described by the Hamiltonian $H_{S_{m}^{q}}$ on $\mathcal{S} \otimes_{n=0}^{m-1} \mathcal{K}_{n}$,

$$
\begin{aligned}
H_{S_{m}^{q}} & :=H_{S} \otimes \mathbb{I}_{\Gamma(\mathfrak{h})}+\sqrt{\beta_{0}(q)} A \otimes\left(b_{0}^{*}+b_{0}\right) \\
& +\mathbb{I}_{\mathcal{S}} \otimes \sum_{n=0}^{m-2} \sqrt{\beta_{n+1}(q)}\left(q\left(b_{n}^{*} b_{n+1}^{*}+b_{n} b_{n+1}\right)+\left(b_{n}^{*} b_{n+1}+b_{n} b_{n+1}^{*}\right)\right) \\
& +\mathbb{I}_{\mathcal{S}} \otimes \sum_{n=0}^{m-1}\left(\frac{q}{2} \alpha_{n}(q)-\frac{q^{2}}{8}\right)\left(b_{n}^{*} b_{n}^{*}+b_{n} b_{n}\right)+\left(\alpha_{n}(q)+\frac{q}{4}\right) b_{n}^{*} b_{n} .
\end{aligned}
$$

The mth environment is formed by the remaining environment terms in the Hamilitonian, in other words the quantum system described by the Hamiltonian $H_{E_{m}^{q}}$ on $\otimes_{n=m}^{\infty} \mathcal{K}_{n}$,

$$
\begin{aligned}
H_{E_{m}^{q}} & =\frac{q}{2}\left[\mathbf{b}_{m}^{T}\left(\mathcal{J}\left(d \lambda_{m}^{q}\right)-\frac{q}{4} \mathbb{I}\right) \mathbf{b}_{m}+h . c .\right]+\mathbf{b}_{m}^{*}\left(\mathcal{J}\left(d \lambda_{m}^{q}\right)+\frac{q}{4} \mathbb{I}\right) \mathbf{b}_{m} \\
m & =1,2,3, \ldots
\end{aligned}
$$

Hence we have

$$
H=H_{S_{m}^{q}}+\sqrt{\beta_{m}(q)}\left(q\left(b_{m-1}^{*} b_{m}^{*}+b_{m-1} b_{m}\right)+\left(b_{m-1}^{*} b_{m}+b_{m-1} b_{m}^{*}\right)\right)+H_{E_{m}^{q}} .
$$

See figure (c) for a pictorial representation. As we will see in section 3.4 the chain coefficients converge for a wide range of spectral densities, and hence all the specific features of an environment appear in the first sites of the chain. Consequently, these can be progressively (or directly, all in one go) absorbed into the system by making an embedding; to reduce the complexity of the effective environment.

Definition 20 We call the nth residual spectral density $J_{n}(\omega)$ to the spectral density which describes the system-environment interaction of the nth embedding. We call the initial spectral density $J_{0}(\omega)$ such that $J_{0}(\omega) \equiv J(\omega)$. See figure (c). 
3.2 Connection between the phonon mapping to previous work and the sequence of residual spectral densities

Theorem 18 The sequence of residual gapless spectral densities in 21] are generated by

$$
J_{n}(\omega)=\frac{J_{0}(\omega)}{\left(P_{n-1}\left(d \lambda^{1} ; \omega^{2}\right) \frac{\varphi\left(d \lambda^{1} ; \omega^{2}\right)}{2}-Q_{n-1}\left(d \lambda^{1} ; \omega^{2}\right)\right)^{2}+J_{0}^{2}(\omega) P_{n-1}^{2}\left(d \lambda^{1} ; \omega^{2}\right)}
$$

$n=1,2,3, \ldots$.

Proof As observed by Leggett [39], when the spectral density of an open quantum system has support on a real interval (in contrast to having support on disjoint intervals), one can easily obtain it from it's propagator $L_{0}(z)$. The authors of 21 have developed this to find a continued fraction representation for the case of the mapping of the propagator presented in their paper as follows.

$$
w_{0}(z)=\frac{-z^{2}-w_{0}(z),}{\Omega_{1}^{2}-z^{2}-\frac{D_{0}^{2}}{\Omega_{2}^{2}-z^{2}-\frac{D_{1}^{2}}{\Omega_{3}^{2}-z^{2}-\ldots}}},
$$

where

$$
\begin{aligned}
D_{n}^{2} & =\frac{2}{\pi} \int_{0}^{\infty} d \omega J_{n}(\omega) \omega \quad n=0,1,2, \ldots, \\
\Omega_{n+1}^{2} & =\frac{2}{\pi D_{n}^{2}} \int_{0}^{\infty} d \omega J_{n}(\omega) \omega^{3} \quad n=0,1,2, \ldots,
\end{aligned}
$$

and $z \in \mathbb{C}-\operatorname{support}\left(J_{0}\right)$, where support $\left(J_{0}\right)$ is an interval on the real line by definition [21. Alternatively, we note that we can write the continued fraction Eq. (186) as a recurrence relation

$$
w_{n}^{\prime}(\sqrt{z})=\frac{D_{n}^{2}}{z-\Omega_{n+1}^{2}-w_{n+1}^{\prime}(\sqrt{z})} \quad n=0,1,2, \ldots,
$$

where $w_{n}^{\prime}(z):=-w_{n}(z)$. From [21, we have an alternative expression for $w_{n}$ in terms of the residual spectral densities though the relation,

$$
w_{n}(z)=\frac{2}{\pi} \int_{0}^{\infty} d \omega \frac{J_{n}(\omega) \omega}{\omega^{2}-z^{2}} \quad n=0,1,2, \ldots,
$$

which by a change of variables and taking into account the definition of $\omega_{n}^{\prime}$ in Eq. (189), can be written in the form

$$
w_{n}^{\prime}(\sqrt{z})=\frac{1}{\pi} \int_{0}^{\infty} d \omega \frac{J_{n}(\sqrt{\omega})}{z-\omega} \quad n=0,1,2, \ldots
$$


Furthermore, via a change of variables the integrals Eq. (187) and (188) can be written as

$$
\begin{aligned}
D_{n}^{2} & =\frac{1}{\pi} \int_{0}^{\infty} d \omega J_{n}(\sqrt{\omega}) \quad n=0,1,2, \ldots, \\
\Omega_{n+1}^{2} & =\frac{1}{\pi D_{n}^{2}} \int_{0}^{\infty} d \omega J_{n}(\sqrt{\omega}) \omega \quad n=0,1,2, \ldots .
\end{aligned}
$$

We note that in 21] the support of the spectral densities corresponds with the domain of the spectral densities defined in definition (16) and hence integrals Eq. (191), (192) and (193) are zero outside of the domain of $J_{n}(x), \quad n=0,1,2, \ldots$, therefore we can change the lower limit of 0 and upper limit of $\infty$ of the integrals by $\omega_{\min }^{2}$ and $\omega_{\max }^{2}$ respectively. Now let us define the set of measures

$$
\begin{aligned}
d \gamma_{n}(t) & =d t \bar{\gamma}_{n}(t) \quad n=0,1,2, \ldots \\
\bar{\gamma}_{n}(t) & =\frac{J_{n}(\sqrt{t})}{\pi D_{n}^{2}} \quad n=0,1,2, \ldots
\end{aligned}
$$

This definition of the measure has some important consequences:

1) From Eq. (193) we note that $\Omega_{n+1}^{2}$ are the first moments of the measures $d \gamma_{n}(t)$,

$$
C_{1}\left(d \gamma_{n}\right)=\Omega_{n+1}^{2} \quad n=0,1,2, \ldots
$$

2) From Eq. (191) and definition (7) we see that $w_{n}^{\prime}(\sqrt{z})$ is proportional to the Stieltjes transformations of the measure $d \gamma_{n}(t)$

$$
w_{n}^{\prime}(\sqrt{z})=D_{n}^{2} S_{n}(z) \quad n=0,1,2, \ldots
$$

3) From Eq. (192) we see that the zeroth moments of the measures $d \gamma_{n}(t)$ are unity

$$
C_{0}\left(d \gamma_{n}\right)=1 \quad n=0,1,2, \ldots
$$

We are now able to re-write Eq. (189) in the form

$$
S_{n+1}(z) D_{n+1}^{2}=z-C_{1}\left(d \gamma_{n}\right)-\frac{1}{S_{n}(z)}, \quad n=0,1,2, \ldots,
$$

where we have used the short hand $S_{m_{n}}(z)=: S_{n}(z)$. By comparing this recursion relation with Eq. (21) and definition (8), we deduce that $D_{n+1}^{2} d \gamma_{n+1}$ is the secondary measure associated with $d \gamma_{n}$, for $n=0,1,2, \ldots$. We can also identify a sequence of normalised secondary measures. Noting that $C_{0}\left(d \gamma_{0}\right)=1$ from Eq. (198), definition (9) tells us that the sequence of secondary normalised measures starting from $d \gamma_{0}$ is

$$
d \gamma_{0}, D_{1}^{2} d \gamma_{1} / C_{0}\left(D_{1}^{2} d \gamma_{1}\right), D_{2}^{2} d \gamma_{2} / C_{0}\left(D_{2}^{2} d \gamma_{2}\right), \ldots, D_{m}^{2} d \gamma_{m} / C_{0}\left(D_{m}^{2} d \gamma_{m}\right), \ldots
$$

However,

$$
C_{0}\left(D_{n}^{2} d \gamma_{n}\right)=D_{n}^{2} C_{0}\left(d \gamma_{n}\right)=D_{n}^{2} \quad n=1,2,3 \ldots
$$


so the sequence of normalised secondary measures is $d \gamma_{0}(t), d \gamma_{1}(t), d \gamma_{2}(t), d \gamma_{3}(t), \ldots$ Taking into account lemma (3) and theorem (8) we see that

$$
D_{n}^{2}=d_{n-1}=\beta_{n}\left(d \gamma_{0}\right) \quad n=1,2,3 \ldots
$$

Due to corollary (11) and Eq. (198) we can also write $D_{0}^{2}$ in terms of $\beta_{0}$,

$$
D_{0}^{2}=\beta_{0}\left(d \eta_{0}\right)
$$

where

$$
d \eta_{0}:=D_{0}^{2} d \gamma_{0}
$$

We can now construct a sequence of beta normalised measures from $d \eta_{0}$, denoted by $d \eta_{0}$, $d \eta_{1}, d \eta_{2}, \ldots$. From Eq. (203) and (204) we see that $d \eta_{0}$ satisfies Eq. (103) for $d \gamma_{0}$, hence lemma (9) tells us

$$
\bar{\eta}_{n}(x)=\beta_{n}\left(d \eta_{0}\right) \bar{\gamma}_{n}(x) \quad n=0,1,2, \ldots .
$$

Taking into account that $\beta_{n}\left(d \eta_{0}\right)=\beta_{n}\left(d \gamma_{0}\right) \quad n=1,2,3, \ldots$ due to lemma (7) and Eq. (204), from Eq. (205) and (195) we gather

$$
\begin{aligned}
& d \eta_{n}(t)=d t \bar{\eta}_{n}(t) n=0,1,2, \ldots \\
& \bar{\eta}_{n}(t)=\frac{J_{n}(\sqrt{t})}{\pi} \quad n=0,1,2, \ldots
\end{aligned}
$$

hence we note that

$$
d \eta_{0}=d \lambda^{1}
$$

Substituting this into Eq. (101) gives us Eq. (184).

In [21, a similar mapping as in theorem (17) is developed. Starting from a Hamiltonian of the form Eq. (119), they show that it is equivalent to a Hamiltonian of the form

$$
H_{S}-D_{0} s \otimes X_{1}^{\prime}+\mathbb{I}_{\mathcal{S}} \otimes \sum_{n=1}^{\infty}\left(-D_{n} X_{n}^{\prime} X_{n+1}^{\prime}+\frac{\Omega_{n}^{2}}{2} X_{n}^{\prime 2}+\frac{1}{2} P_{n}^{\prime 2}\right),
$$

where $H_{S}=\frac{P^{2}}{2 m}+V(s)+\Delta V(s)$ describes the quantum system dynamics and the selfadjoint operator $s$ couples the quantum system to the bosonic bath. The domain of the operators $\left\{X_{n}^{\prime}, P_{n}^{\prime}\right\}_{n=1}^{\infty}$ is not defined by the authors nor the precise relation to the operators in the initial Hamiltonian. See [21] for more details. Here $X_{n}$ and $P_{n}$ are position and momentum operators satisfying $\left[X_{n}, P_{m}\right]=i \delta_{n, m}$. The derivation of the coefficients $D_{n}$, $\Omega_{n}$ involve repeated integration and apriori, seem unrelated to our method. However, given the apparent similarity between Eqs. (209) and (170), a more detailed analysis using some of the theorems developed in section 2 allows one to derive the following theorem which demonstrates that the the mapping of [21] is a special case of the phonon mapping, i.e. it is essentially the same as the phonon mapping for the case of a gappless spectral density.

\footnotetext{
${ }^{8}$ We say "of the form" because the authors do not define the hamiltonian rigorously.
} 
Theorem 19 Define $X_{n}^{\prime}:=(-1)^{n} X_{n-1}, P_{n}^{\prime}=(-1)^{n} P_{n-1}, s=A, \mathcal{D}\left(X_{n}^{\prime}\right)=\mathcal{D}\left(X_{n}\right)$, $\mathcal{D}\left(P_{n}^{\prime}\right)=\mathcal{D}\left(P_{n}\right), \mathcal{D}(s)=\mathcal{D}(A), n=1,2,3, \ldots$, and let the spectral density $J_{0}$ be gapless, then

$$
\begin{aligned}
& -D_{0} s \otimes X_{1}^{\prime}+\mathbb{I}_{\mathcal{S}} \otimes \sum_{n=1}^{\infty}\left(-D_{n} X_{n}^{\prime} X_{n+1}^{\prime}+\frac{\Omega_{n}^{2}}{2} X_{n}^{\prime 2}+\frac{1}{2} P_{n}^{\prime 2}\right)= \\
& \sqrt{\beta_{0}(1)} A \otimes X_{0}+\mathbb{I}_{\mathcal{S}} \otimes \sum_{n=0}^{\infty}\left(\sqrt{\beta_{n+1}(1)} X_{n} X_{n+1}+\frac{\alpha_{n}(1)}{2} X_{n}^{2}+\frac{1}{2} P_{n}^{2}\right) .
\end{aligned}
$$

Proof From Eq. (193) and (91) we see that we can write $\Omega_{n}^{2}$ as

$$
\Omega_{n+1}^{2}=\alpha_{0}\left(d \eta_{n}\right) \quad n=0,1,2, \ldots
$$

Hence,

$$
\Omega_{n+1}^{2}=\alpha_{n}\left(d \eta_{0}\right)=\alpha_{n}\left(d \lambda^{1}\right)=\alpha_{n}(1) \quad n=0,1,2, \ldots,
$$

where we have used Eq. (110) followed by Eq. (208). From Eq. (192), and (11) we see that

$$
D_{n}^{2}=\beta_{0}\left(d \eta_{n}\right) \quad n=0,1,2, \ldots
$$

Hence using Eq. (111) followed by Eq. (208), we find

$$
D_{n}^{2}=\beta_{n}\left(d \eta_{0}\right)=\beta_{n}\left(d \lambda^{1}\right)=\beta_{n}(1) \quad n=0,1,2, \ldots
$$

Now using the definitions stated in the theorem, we conclude the proof.

Remark 7 We note that the three term recurrence relations Eq. (7) for gapped measures, still hold. Hence the generalised mapping (and therefore the phonon mapping) is still valid. However, the chain mapping presented in 21] is not valid under these conditions because the relation between the Stieltjes transformations of two consecutive measures, Eq. (21) is not valid anymore (see (6.1) in appendix for a proof) and hence one cannot calculate the chain coefficients from the sequence of residual spectral densities. In this sense, the phonon mapping presented here is a more general result. This is an important difference because if a spectral density is gapped, then the corresponding measure is also gapped. There are physical systems (such as photonic crystals and diatomic chains) which have these properties.

Corollary 8 In the phonon mapping case, if the spectral density is gapless the sequence of residual spectral densities is given by

$$
J_{n}(\omega)=\frac{J_{0}(\omega)}{\left(P_{n-1}\left(d \lambda^{1} ; \omega^{2}\right) \frac{\varphi\left(d \lambda^{1} ; \omega^{2}\right)}{2}-Q_{n-1}\left(d \lambda^{1} ; \omega^{2}\right)\right)^{2}+J_{0}^{2}(\omega) P_{n-1}^{2}\left(d \lambda^{1} ; \omega^{2}\right)}
$$

$n=1,2,3, \ldots$

Proof Follows directly from theorems (18) and (19). Alternatively, we note that by applying the same line of reasoning of the proof for theorem (20) to the phonon mapping Hamiltonian Eq. (170), we can easily provide an alternative proof for this corollary. This has the advantage of being an independent derivation from the results of [21, but has the downside of not illustrating the connections between this paper and their results. 
3.3 Sequence of residual spectral densities for the particle mapping case

Theorem 20 In the particle mapping case, if the spectral density is gapless the sequence of residual spectral densities is given by

$$
J_{n}(\omega)=\frac{J_{0}(\omega)}{\left(P_{n-1}\left(d \lambda^{0} ; \omega\right) \frac{\varphi\left(d \lambda^{0} ; \omega\right)}{2}-Q_{n-1}\left(d \lambda^{0} ; \omega\right)\right)^{2}+J_{0}^{2}(\omega) P_{n-1}^{2}\left(d \lambda^{0} ; \omega\right)}
$$

$n=1,2,3, \ldots$

Proof The proof will start by defining a set of initially independent Hamiltonians. Each one of these will represent some system-environment interaction of a similar type discussed in this paper. When they are in this form, we can easily find an expression for the spectral density representing the system-environment interaction. We then put constrains on the Hamiltonians in such a way that they are no-longer independent from one another. Finally we show that these spectral densities correspond to the sequence of spectral densities for the particle mapping.

Let us start by defining a set of independent Hamiltonians on $\mathcal{H}$ labelled by $m$

$H_{m}=H_{S_{m}^{0}}+\mathbb{I}_{\mathcal{S}} \otimes \int_{k_{m i n}}^{k_{\max }} g(x) a_{x, m}^{*} a_{x, m} d x+\mathbb{I}_{\mathcal{S}} \otimes \int_{k_{m i n}}^{k_{\max }} h_{m}(x)\left(a_{x, m}^{*} b_{m-1}(0)+a_{x, m} b_{m-1}^{*}(0)\right) d x$

$m=1,2,3 \ldots$, where $H_{S_{m}^{0}}$ is defined in (178), the creation $b_{m}^{*}(0)$ annihilation $b_{m}(0)$ operators in theorem (17) and the creation $a_{k, m}^{*}$, annihilation $a_{k, m}$ satisfy $\left[a_{k, m}, a_{k^{\prime}, m}^{*}\right]=\delta\left(k-k^{\prime}\right)$ and will be defined later in the proof in terms of $b_{m}^{*}(0), b_{m}(0)$. For the particular choice of the functions $\left\{h_{m}\right\}_{m=1}^{\infty}$ that we will make in the proof, we will show that the $H_{m}$ are equal to the Hamiltonian (165), and hence self-adjoint on $\mathcal{H}$. We note that the $m$ th Hamitonian $H_{m}$, has a spectral density $J^{m}(x)$ given by $\mathrm{Eq}$. (127):

$$
J^{m}(\omega)=\pi h_{m}^{2}\left[g^{-1}(\omega)\right]\left|\frac{d g^{-1}(\omega)}{d \omega}\right|
$$

Eq. (219) gives us $h_{m}^{2}(x)=J^{m}(g(x))|d g(x) / d x| / \pi$. We now define the set of measures

$$
d \vartheta_{m}(x)=\frac{J^{m}(g(x))}{\pi} d x \quad m=1,2,3, \ldots,
$$

and the new set of creation and annihilation operators

$$
\begin{aligned}
b_{n+m}^{*} & =\int_{k_{\min }}^{k_{\max }} d x U_{n}^{m}(x) a_{x, m}^{*}, \\
b_{n+m} & =\int_{k_{\min }}^{k_{\max }} d x U_{n}^{m}(x) a_{x, m} \quad n=0,1,2, \ldots, m=1,2,3, \ldots,
\end{aligned}
$$

\footnotetext{
${ }^{9}$ We have used a superindex here rather than a subindex to denote the spectral densities so as not to confuse them with residual spectral densities, as at this stage we cannot identify them as such.
} 
where $U_{n}^{m}(x)=h_{m}(x) P_{n}\left(d \vartheta_{m} ; g(x)\right) \quad n=0,1,2, \ldots, m=1,2,3, \ldots$. Substituting the inverse relations

$$
\begin{aligned}
a_{x, m}^{*} & =\sum_{n=0}^{\infty} U_{n}^{m}(x) b_{n+m}^{*}, \\
a_{x, m} & =\sum_{n=0}^{\infty} U_{n}^{m}(x) b_{n+m} \quad n=0,1,2, \ldots, m=1,2,3, \ldots,
\end{aligned}
$$

into the RHS of Eq. (218) and using the three term recurrence relations and orthogonality conditions in Lemma (10) for $q=0$, we find that we can write $H_{m}$ as

$$
\begin{aligned}
H_{m} & =H_{S m}+\sqrt{\beta_{0}\left(d \vartheta_{m}\right)}\left(b_{m}^{*} b_{m-1}+b_{m} b_{m-1}^{*}\right) \\
& +\sum_{n=0}^{\infty} \sqrt{\beta_{n+m+1}\left(d \vartheta_{m}\right)}\left(b_{n+m+1}^{*} b_{n+m}+\text { h.c. }\right)+\alpha_{n}\left(d \vartheta_{m}\right) b_{n+m}^{*} b_{n+m}
\end{aligned}
$$

$m=1,2,3, \ldots$. We note that at this stage, the set of spectral densities $\left\{J^{m}(x)\right\}_{m=1}^{\infty}$ are independent and undefined. This freedom allows us to let the set of measures $\left\{d \vartheta_{m}\right\}_{m=1}^{\infty}$ be a sequence of beta normalised measures generated from the measure $d \lambda^{0}(x)=M^{0}(x) d x$ which from Eq. (166) we see is given in terms of another spectral density $J(x)$. Now the spectral densities $\left\{J^{m}(x)\right\}_{m=1}^{\infty}$ are fully determined by $J(x)$ through the definition of a beta normalised sequence of measures, definition (13). Hence using Eq. (101) and (220) we find

$$
J^{n}(\omega)=\frac{J(\omega)}{\left(P_{n-1}\left(d \lambda^{0} ; \omega\right) \frac{\varphi\left(d \lambda^{0} ; \omega\right)}{2}-Q_{n-1}\left(d \lambda^{0} ; \omega\right)\right)^{2}+J^{2}(\omega) P_{n-1}^{2}\left(d \lambda^{0} ; \omega\right)}
$$

$n=1,2,3, \ldots$. Now we have to show that $J^{n}$ is the nth residual spectral density for the particle mapping Hamiltonian Eq. (165) i.e. the interaction described by Eq. (183) when $q=0$. First note that using Eq. (110) and (111), we have

$$
\begin{aligned}
\beta_{0}\left(d \vartheta_{m}\right) & =\beta_{m}\left(d \lambda^{0}\right) \quad m=1,2,3, \ldots, \\
\alpha_{n}\left(d \vartheta_{m}\right) & =\alpha_{n+m}\left(d \lambda^{0}\right) \quad m=1,2,3, \ldots, n=0,1,2, \ldots, \\
\beta_{n+1}\left(d \vartheta_{m}\right) & =\beta_{n+1+m}\left(d \lambda^{0}\right) \quad m=1,2,3, \ldots, n=0,1,2, \ldots
\end{aligned}
$$

Substituting these identities into Eq. (225), we see that all $\left\{H_{m}\right\}_{m=1}^{\infty}$ are equal to one another and equal to (165).

\subsection{Residual spectral densities sequence convergence}

Definition 21 We say that the chain mapping for some $q$ and a particular spectral density $J(x)$, will belong to the Szegö class if the measure $d \lambda^{q}(x)=M^{q}(x) d x$ satisfies

$$
\int_{G_{q}\left(\omega_{\min }\right)}^{G_{q}\left(\omega_{\max }\right)} \frac{\ln M^{q}(x) d x}{\sqrt{\left(G_{q}\left(\omega_{\max }\right)-x\right)\left(x-G_{q}\left(\omega_{\min }\right)\right)}}>-\infty .
$$

Remark 8 Examples of spectral densities which for any $q$ do not belong to the Szegö class, are those which are gapped and those with unbounded support. 
Theorem 21 If for some $q$ and spectral density $J(\omega)$, chain mapping belongs to the Szegö class, then the sequences $\alpha_{0}(q), \alpha_{1}(q), \alpha_{2}(q), \ldots$ and $\beta_{0}(q), \beta_{1}(q), \beta_{2}(q), \ldots$ converge to:

$$
\begin{aligned}
& \lim _{n \rightarrow \infty} \alpha_{n}(q)=\frac{G_{q}\left(\omega_{\max }\right)+G_{q}\left(\omega_{\min }\right)}{2}, \\
& \lim _{n \rightarrow \infty} \beta_{n}(q)=\frac{\left(G_{q}\left(\omega_{\max }\right)-G_{q}\left(\omega_{\min }\right)\right)^{2}}{16} .
\end{aligned}
$$

Proof Follows from shifting the support region of the Szegö theorem in [18]. For the original theorem see [40].

Corollary 9 If for some $q$ and spectral density $J(\omega)$ the chain mapping belongs to the Szegö class, the tail of the semi-infinite chain mapping tends to a translational invariant chain. In other words,

$$
\lim _{n \rightarrow \infty} E_{p, n}(q)=C_{p}(q) \quad p=1, \ldots, 5,
$$

where $C_{p} \in \mathbb{R}$ are finite constants for all constant $q \in[0,1]$ and $E_{p, n}$ are defined in theorem (17).

Proof Follows from theorem (21) and Eq. (163).

Definition 22 The moment problem for a measure $d \mu$ is said to be determined, if it is uniquely determined by its moments.

Theorem 22 If a measure $d \mu$ has a finite support interval I, then its moment problem is determined.

Proof See [27.

Theorem 23 If for some gappless measure $d \mu(x)$ with finite support interval I the limits

$$
\begin{aligned}
& \lim _{n \rightarrow \infty} \alpha_{n}(d \mu)=\frac{a+b}{2}, \\
& \lim _{n \rightarrow \infty} \beta_{n}(d \mu)=\frac{(b-a)^{2}}{16},
\end{aligned}
$$

exist, then the sequence of beta normalised and normalised secondary measures generated from $d \nu$ and $d \mu$ respectively, converge weakly to

$$
\begin{aligned}
& \lim _{n \rightarrow \infty} \bar{\nu}_{n}(x)=\frac{\sqrt{(x-a)(b-x)}}{2 \pi}, \\
& \lim _{n \rightarrow \infty} \bar{\mu}_{n}(x)=\frac{8 \sqrt{(x-a)(b-x)}}{\pi(b-a)^{2}} .
\end{aligned}
$$

Proof First we will show that the limit exists by construction (this is to say, by finding the fixed points of the sequence), then we will show that it corresponds to when the limits Eq. (235) and (236) are accomplished.

Substituting Eq. (62) into Eq. (56), we find

$$
\varphi(d \rho ; x)=2\left[x-C_{1}(d \mu)\right]-\frac{\varphi(d \mu ; x) \bar{\rho}(x)}{\bar{\mu}(x)} .
$$


If the limit exists, then there must be at least one solution to $\bar{\rho}(x)=A \bar{\mu}(x)$ where $d \rho(x)=$ $\bar{\rho}(x) d x$ is the secondary measure associated with the gapless measure $d \mu(x)=\bar{\mu}(x) d x$ and $A>0$. From definitions (11) and (7) we see that $\varphi(d \rho ; x)=A \varphi(d \mu ; x)$ and hence from Eq. (239) we see that

$$
\varphi(d \mu ; x)=\frac{x-C_{1}(d \mu)}{A} .
$$

Thus substituting this into Eq. (馬), and solving for $\bar{\mu}(x)$ we find

$$
\bar{\mu}^{2}(x)=\frac{4 A-\left(x-C_{1}(d \mu)\right)^{2}}{4 \pi^{2} A^{2}} .
$$

Taking into account the definition of a gapless measure, definition (1), we see that if the limit exits, then it must be bounded. Moreover, it must belong to the interval centered at $C_{1}(d \mu)$ and of length $4 \sqrt{A}$. Making the change of variable $x-C_{1}(d \mu)=2 \sqrt{A} t$ gives us

$$
d \mu(t)=\bar{\mu}(t) d t=\frac{2 \sqrt{1-t^{2}}}{\pi} d t
$$

with support interval $[-1,1]$. We now can check that Eq. 242 exists by direct substitution. Using definition (7) we find that the Stieltjes Transform of Eq. (242) is $S_{\bar{\mu}}(z)=2[z-$ $\sqrt{z^{2}-1}$ ] and hence from definition (11) we find $\varphi(d \mu ; x)=4 x$. Thus using Eq. (56) we have $\bar{\rho}(x)=\bar{\mu}(x) / 4$, hence the limit exists. By performing the change of variable $t=$ $(2 /(b-a)) y+(b+a) /(a-b)$, we shift the support of Eq. (242) to the general case $[a, b]$ and the measure is now given by Eq. (238).

Now we will proceed to show that if Eq. (235) and (236) are satisfied, then the sequence of normalised secondary measures converge weakly to Eq. (238). By taking the nth moment of the measures in Eq. (24) and taking into account lemma (26) for $n=0$, we have

$$
C_{n}\left(d \rho_{n+1}\right)=\left[C_{2}\left(d \mu_{n}\right)-C_{1}\left(d \mu_{n}\right)^{2}\right] C_{n}\left(d \mu_{n+1}\right) \quad n, m=0,1,2, \ldots
$$

Writing Eq. (26) for a sequence of measures followed by substituting in our expression for $C_{n}\left(d \rho_{n+1}\right)$ using Eq. (243), we find

$$
\left(c_{2}^{s}-\left(c_{1}^{s}\right)^{2}\right) c_{n}^{s+1}=c_{n+2}^{s}-c_{1}^{s} c_{n+1}^{s}-\sum_{j=0}^{n-1}\left(c_{2}^{s}-\left(c_{1}^{s}\right)^{2}\right) c_{j}^{s+1} c_{n-j}^{s} \quad n, s=0,1,2, \ldots
$$

where $c_{n}^{s}:=C_{n}\left(d \mu_{s}\right) \quad n, s=0,1,2, \ldots$ Let us define the limit $l_{n}=\lim _{s \rightarrow \infty} c_{n}^{s}$. We can now draw the following conclusions

1) Due to corollary (2), we have $C_{0}\left(d \mu_{n}\right)=1 \quad n=0,1,2, \ldots$ Therefore $l_{0}=1$.

2) From Eq. (89) and (9) we see that

$$
\alpha_{n}\left(d \mu_{0}\right)=\alpha_{0}\left(d \mu_{n}\right)=\frac{C_{1}\left(d \mu_{n}\right)}{C_{0}\left(d \mu_{n}\right)}=c_{1}^{n}
$$

Hence taking into account assumption Eq. (235), we have $l_{1}=(a+b) / 2$. 
3) Noting the definition of $d_{n}$ in theorem (3), theorem (8) tells us

$$
\beta_{n+1}\left(d \mu_{0}\right)=c_{2}^{n}-\left(c_{1}^{n}\right)^{2} \quad n=0,1,2, \ldots .
$$

Hence taking into account assumptions Eq. (235) and (236), and Eq. (245) we have $l_{2}=\left(5 a^{2}+6 a b+5 b^{2}\right) / 16$.

We note that for any $s$, all the moments $c_{n}^{s}$ in Eq. (244) are fully determined by the starting values $c_{0}^{s}, c_{1}^{s}$, and $c_{2}^{s}$, hence we conclude from the above points 1 ), 2) and 3 ), that under the assumptions Eq. (235) and (236), all $l_{n} \quad n=0,1,2, \ldots$ are finite and determined by

$$
\left(l_{2}-l_{1}^{2}\right) l_{n}=l_{n+2}-l_{1} l_{n+1}-\left(l_{2}-l_{1}^{2}\right) \sum_{j=0}^{n-1} l_{j} l_{n-j} \quad n, s=0,1,2, \ldots,
$$

with starting values $l_{0}=1, l_{1}=(a+b) / 2$, and $l_{2}=\left(5 a^{2}+6 a b+5 b^{2}\right) / 16$.

For the case of the normalised measure Eq. (238), we conclude that $c_{n}^{s}=c_{n}^{s+1} n, s=$ $0,1,2, \ldots$ since its secondary normalised measure is equal to itself. Hence by denoting $m_{n}=$ $c_{n}^{s} \quad n, s=0,1,2, \ldots$ we can write Eq. (244) for this measure as

$$
\left(m_{2}-\left(m_{1}\right)^{2}\right) m_{n}=m_{n+2}-m_{1} m_{n+1}-\left(m_{2}-\left(m_{1}\right)^{2}\right) \sum_{j=0}^{n-1} m_{j} m_{n-j}
$$

$n, s=0,1,2, \ldots$ By direct calculation of the moments of Eq. (238), we find that $m_{0}=$ $l_{0}, m_{1}=l_{1}, m_{2}=l_{2}$, and hence by comparing Eq. (247) with Eq. (248) we conclude that $m_{n}=l_{n} \quad n=0,1,2, \ldots$. Thus using lemma (22) we conclude Eq. (238) under the assumptions Eq. (235) and (236). For Eq. (237), we note that Eq. (102) and Eq. (107) tell us $\lim _{n \rightarrow \infty} \bar{\nu}_{n}(x)=\lim _{n \rightarrow \infty} \beta_{n}\left(d \mu_{0}\right) \bar{\mu}_{n}(x)$. Hence from Eq. (236) and (238) we conclude Eq. (237).

Definition 23 We will call terminal spectral density $J_{T}(\omega)$ the spectral density to which a sequence of residual spectral densities converge to weakly if such a limit exists: $J_{T}(\omega)=$ $\lim _{n \rightarrow \infty} J_{n}(\omega)$.

We are now ready to state our fourth main theorem:

Theorem 24 If for the particle or phonon mapping the spectral density $J(\omega)$ belongs to the Szegö class, the sequence of residual spectral densities converge weakly to the Wigner semicircle distribution 41.

$$
J_{T}(\omega)=\frac{\sqrt{\left(\omega-\omega_{\min }\right)\left(\omega_{\max }-\omega\right)}}{2},
$$

and the Rubin model spectral density [1]

$$
J_{T}(\omega)=\frac{\sqrt{\left(\omega^{2}-\omega_{\min }^{2}\right)\left(\omega_{\max }^{2}-\omega^{2}\right)}}{2},
$$

respectively, $\omega \in\left[\omega_{\min }, \omega_{\max }\right]$. 
Proof From Eq. (207) and theorem (23) we gather that if Eq. (235) and (236) are satisfied, then for the phonon case

$$
J_{T}(\omega)=\frac{\sqrt{\left(\omega^{2}-a\right)\left(b-\omega^{2}\right)}}{2} .
$$

From Eq. (172) we have that $a=\omega_{\text {min }}^{2}$ and $b=\omega_{\text {max }}^{2}$. Now taking into account theorem (21), we find Eq. (250). Proceeding in a similar manner, we find Eq. (249).

\section{Examples}

4.1 power law spectral densities with finite support

The widely studied power law spectral densities are [10, 1]

$$
J(x)=2 \pi \alpha \omega_{c}^{1-s} x^{s},
$$

with domain $\left[0, \omega_{c}\right]$ and $s>-1$. Let us start by calculating the sequence of residual spectral densities for the case of the particle mapping.

From Eq. (166) and (252) we have

$$
M^{0}(x)=2 \alpha \omega_{c}^{1-s} x^{s}
$$

For simplicity, we will scale out the $\omega_{c}$ dependency and show how to put it back again afterwards. Let us start by defining the weight function $m_{0}^{0}(x):=\omega_{c} M^{0}\left(x \omega_{c}\right)=2 \alpha \omega_{0}^{2} x^{s}$ with support interval $[0,1]$. From lemma (12), we find that $m_{n}^{0}(x)=M_{n}^{0}\left(x \omega_{c}\right) / \omega_{c} \quad n=$ $1,2,3, \ldots$ where $m_{n}^{0}(x)$ and $M_{n}^{0}(x)$ are the sequence of beta normalised measures generated from $m_{0}^{0}(x)$ and $M^{0}(x)$ respectively. Now let us define the weight function

$$
\tilde{m}_{0}^{0}(x)=x^{s}
$$

with support interval $[0,1]$. Given that this new measure is proportional to $m_{0}^{0}(x)$, from lemma (13) we conclude that it's sequence of beta normalised measures $\tilde{m}_{n}^{0}(x) \quad n=$ $1,2,3, \ldots$, are equal. Hence we have $M_{n}^{0}(x)=\omega_{c} \tilde{m}_{n}^{0}\left(x / \omega_{c}\right) \quad n=1,2,3, \ldots$. Thus taking into account Eq. (166) and (220), we conclude

$$
J_{n}(\omega)=\pi M_{n}^{0}(\omega)=\omega_{c} \pi \tilde{m}_{n}^{0}\left(\omega / \omega_{c}\right) \quad n=1,2,3, \ldots
$$

The real polynomials orthogonal to the weight function $\tilde{m}_{0}^{0}(x)$ are $P_{n}^{s}(x):=P_{n}^{(0, s)}(2 x-$ 1) $\sqrt{n+s+1} n=0,1,2, \ldots$; which are normalised shifted counterparts of the Jacobi polynomials $P_{n}^{(\alpha, \beta)}(x) \quad n=0,1,2, \ldots$. The reducer for the case $s \geq 0$ is given by theorem (26)

$$
\varphi\left(d \tilde{m}^{0} ; t\right)=2\left[\ln \left(\frac{t}{1-t}\right)+s \int_{0}^{1} x^{s-1} \ln \left|\frac{t-x}{t}\right| d x\right]
$$


which has analytic solutions when a particular value of $s$ is specified. For example, the first three in the sequence for the ohmic case $(s=1)$ are

$$
\begin{aligned}
& \tilde{m}_{1}^{0}(x)=\frac{x}{2\left(\pi^{2} x^{2}+\left[1+x \ln \left(\frac{1-x}{x}\right)\right]^{2}\right)}, \\
& \tilde{m}_{2}^{0}(x)=\frac{x}{4 \pi^{2}(2-3 x)^{2} x^{2}+\left[1-6 x+(4-6 x) x \ln \left(\frac{1-x}{x}\right)\right]^{2}} \\
& \tilde{m}_{3}^{0}(x)=\frac{6 x}{36 \pi^{2} x^{2}\left(3-12 x+10 x^{2}\right)^{2}+\left[30 x-16+\left(18-72 x+60 x^{2}\right)\left(1+x \ln \left(\frac{1-x}{x}\right)\right)^{2}\right.} .
\end{aligned}
$$

The chain coefficients are calculated in 18 to be

$$
\begin{gathered}
\alpha_{n}(0)(s)=\frac{\omega_{c}}{2}\left(1+\frac{s^{2}}{(s+2 n)(2+s+2 n)}\right) \quad n=0,1,2, \ldots, \\
\sqrt{\beta_{n+1}(0)}(s)=\frac{\omega_{c}(1+n)(1+s+n)}{(s+2+2 n)(3+s+2 n)} \sqrt{\frac{3+s+2 n}{1+s+2 n}} \quad n=0,1,2, \ldots,
\end{gathered}
$$

where the $(s)$ is to remind us of their $s$ dependency. System environment coupling coefficient is 18 ]

$$
\sqrt{\beta_{0}(0)}=\omega_{c} \sqrt{\frac{2 \alpha}{s+1}} .
$$

Now we will find the sequence of residual spectral densities for the case of the phonon mapping. From Eq. (171) and (252) we have

$$
M^{1}(x)=2 \alpha \omega_{c}^{1-s} x^{s / 2}
$$

with support $\left[0, \omega_{c}^{2}\right]$. Proceeding as in the particle mapping case and taking into account Eq. (207), we find

$$
J_{n}(\omega)=\pi M_{n}^{1}\left(\omega^{2}\right)=\omega_{c}^{2} \pi \tilde{m}_{n}^{1}\left(\omega^{2} / \omega_{c}^{2}\right) \quad n=1,2,3, \ldots,
$$

where $\tilde{m}_{n}^{1}(x) \quad n=1,2,3, \ldots$, are the sequence of beta normalised measures generated from

$$
\tilde{m}_{0}^{1}(x)=x^{s / 2},
$$

with support $[0,1]$. Let us denote $\tilde{m}_{0}^{1}(x)$ and $\tilde{m}_{0}^{0}(x)$ by $\tilde{m}_{0 s}^{1}(x)$ and $\tilde{m}_{0 s}^{0}(x)$ respectively to remind us of their $s$ dependency. By comparing eq. (265) with eq. (254), we have that $\tilde{m}_{0 s}^{1}(x)=\tilde{m}_{0 s / 2}^{0}(x)$ for all $s>-1$ and hence from Eq. (255) and (264) we see that for the Spin-Boson models, there is a simple relationship between the residual spectral densities of the particle and phonon mappings for different $s$ values. For the same example as in the particle case $(s=1)$, we need to evaluate $m_{n}^{0}(x) n=1,2,3, \ldots$, for $s=1 / 2$. The first one in the sequence is

$$
\tilde{m}_{1}^{1}(x)=\frac{2 \sqrt{x}}{3\left(\pi^{2} x+\left(2-2 \sqrt{x} \tanh ^{-1}(\sqrt{x})\right)^{2}\right)},
$$


where $\tanh ^{-1}(x)$ is the inverse hyperbolic tangent function. We can readily calculate the chain coefficients from the particle example. By comparing the expression for the $\alpha_{n}$ and $\beta_{n}$ coefficients for the weight functions for the particle and phonon mappings, we find

$$
\begin{aligned}
\alpha_{n}(1)(s) & =\omega_{c} \alpha_{n}(0)(s / 2) \quad n=0,1,2, \ldots, \\
\sqrt{\beta_{n+1}(1)}(s) & =\omega_{c} \sqrt{\beta_{n+1}(0)}(s / 2) \quad n=0,1,2, \ldots,
\end{aligned}
$$

and system environment coupling term to be

$$
\sqrt{\beta_{0}(1)}=2 \omega_{c} \sqrt{\frac{\alpha \omega_{c}}{s+2}}
$$

For both the particle and phonon mappings, it is easy to verify that the chain coefficients and sequence of residual spectral densities will converge because Eq. (231) is satisfied in both cases as long as $s<\infty$. We also see that the sequence of residual spectral densities calculated in the above examples converge very rapidly to this limit after about the 3rd residual spectral density.

\subsection{The power law spectral densities with exponential cut off}

The power law spectral densities with exponential cut off is [10, 1]

$$
J(x)=2 \pi \alpha \omega_{c}^{1-s} x^{s} e^{-x / \omega_{c}},
$$

with domain $[0, \infty)$ and $s>-1$. Let us start by calculating the sequence of residual spectral densities for the case of the particle mapping.

From Eq. (166) and (270) we have

$$
M^{0}(x)=2 \alpha \omega_{c}^{1-s} x^{s} e^{-x / \omega_{c}} .
$$

Let us define the measure $m^{0}(x):=\omega_{c} M^{0}\left(x \omega_{c}\right)=2 \alpha \omega_{0}^{2} x^{s} e^{-x}$ with support interval $[0, \infty)$. From lemma (12), we find that $m_{n}^{0}(x)=M_{n}^{0}\left(x \omega_{c}\right) / \omega_{c} \quad n=1,2,3, \ldots$ where $m_{n}^{0}(x)$ and $M_{n}^{0}(x)$ are the sequence of beta normalised measures generated from $m^{0}(x)$ and $M^{0}(x)$ respectively. We will now define a 3rd measure by $\tilde{m}^{0}(x)=x^{s} e^{-x}$. We note that it is proportional to the measure $m^{0}(x)$ and hence lemma (13) tells us that its sequence of beta normalised measures are equal. Thus we have the relation

$$
M_{n}^{0}(x)=\omega_{c} \tilde{m}_{n}^{0}\left(x / \omega_{c}\right) \quad n=1,2,3, \ldots
$$

The real polynomials orthogonal to the weight function $\bar{m}^{0}(x)$ are called the associated Laguerre polynomials $L_{n}^{s}(x) \quad n=0,1,2, \ldots$. Their normalised counterparts are $P_{n}^{s}(x):=$ $L_{n}^{s}(x) n ! / \Gamma(n+s+1) \quad n=0,1,2, \ldots$. The reducer in this case is given by theorem (26)

$$
\varphi\left(d \tilde{m}^{0} ; x\right)=2 \int_{0}^{+\infty}(s-t) t^{s-1} e^{-t} \ln \left|\frac{t-x}{x}\right| d t,
$$


which has analytic values when a particular value of $s$ is specified. For example, if $s$ is integer, we find

$$
\varphi\left(d \tilde{m}^{0} ; x\right)=2\left[x^{s} e^{-x} \operatorname{Ei}(x)-\sum_{k=0}^{k=s-1}(s-k-1) ! x^{k}\right]
$$

where $\operatorname{Ei}(x)$ is the exponential integral function 42 .

From Eq. (220) followed by Eq. (272) we have

$$
J_{n}(\omega)=\pi M_{n}^{0}(\omega)=\omega_{c} \pi \tilde{m}_{n}^{0}\left(\omega / \omega_{c}\right) \quad n=1,2,3, \ldots,
$$

and Eq. (101) tells us

$$
\tilde{m}_{n}^{0}(x)=\frac{x^{s} e^{-x}}{\left(P_{n-1}^{s}(x) \frac{\varphi\left(d \tilde{m}^{0} ; x\right)}{2}-Q_{n-1}^{s}(x)\right)^{2}+\pi^{2} x^{2 s} e^{-2 x} P_{n-1}^{s}(x)^{2}} \quad n=1,2,3, \ldots
$$

For example, the first two in the sequence for the ohmic case $(s=1)$ are

$$
\begin{aligned}
& \tilde{m}_{1}^{0}(x)=\frac{x e^{x}}{e^{2 x}+\pi^{2} x^{2}-2 x E i(x) e^{x}+x^{2} E i(x)^{2}}, \\
& \tilde{m}_{2}^{0}(x)=\frac{2 x e^{x}}{e^{2 x}(1-x)^{2}+\pi^{2} x^{2}(x-2)^{2}-2 x\left(2-3 x+x^{2}\right) E i(x) e^{x}+x^{2}(x-2)^{2} E i(x)^{2}} .
\end{aligned}
$$

We also have analytic expressions for the chain coefficients. From [18, we have that the chain coefficients are

$$
\begin{aligned}
\sqrt{\beta_{n+1}(0)} & =\omega_{c} \sqrt{(n+1)(n+s+1)} \quad n=0,1,2, \ldots, \\
\alpha_{n}(0) & =\omega_{c}(2 n+1+s) \quad n=0,1,2, \ldots
\end{aligned}
$$

with the system-environment coupling coefficient given by

$$
\sqrt{\beta_{0}(0)}=\omega_{c} \sqrt{2 \alpha \Gamma(s+1)} .
$$

Similarly, we can calculate the residual spectral densities and chain coefficients for the phonon mapping case.

Because the support interval is infinite, the Spin-Boson model with exponential cut off does not belong to the Szegö class for either the particle mapping nor the phonon mapping as can be easily verified from Eq. (231). This is reflected in the example above as the sequence of residual spectral densities do not converge 10 .

Remark 9 We note that for spectral densities for which the corresponding orthogonal polynomials are unknown analytically, we can easily calculate their coefficients using very stable numerical algorithms. See [19] or [18] and references herein for more details.

\footnotetext{
10 However, we do note that the ratio $\alpha_{n}(q) / \sqrt{\beta_{n+1}(q)}$ for $q=0$ and $q=1$ does converge. This suggests that there is a universal asymptotic expansion for large $n$ for the $n$th residual spectral density.
} 


\section{Summary and conclusion}

By developing the method of [18,19, we have established a general formalism for mapping an open quantum system of arbitrary spectral density of the form Eq. (134) onto chain representations. The different versions of chain mappings are generated by choosing particular values of 4 real constants. This has also provided a very general connection between the theory of open quantum systems and Jacobi operator theory as the semi-infinite chains can be written in terms of Jacobi matrices as can be seen in corollary (7). There has been a wealth of research into the properties of Jacobi operator theory [25], and hence this opens up the theorems of this field to the possibility of being applied to the theory of open quantum systems. Likewise, the new theorem regarding Jacobi matrices (15) developed here, could turn out to be useful in the field of Jacobi operator theory.

There were two previously known exact chain mappings; the one of [18] which is the same as the particle mapping defined here (definition [17), and the mapping by 21. We show that the phonon mapping derived in this article (see definition 18) has a wider range of validity (remark 7) than the mapping of [21 and prove that they are formally equivalent in the range of validity of [21]; see theorem (19).

The concept of embedding degrees of freedom into the system has been around for some time [24,21], however, we see that in chain representations, there is a natural way of shifting the system-environment boundary, that is to say, there is a natural and systematic way of embedding degrees of freedom into the system one by one (or all in one go). To solve quantitatively this problem, we have to first embark on finding the solution to an old problem in mathematics; an analytical solution to the sequence of secondary measures in terms of the initial measure, it's associated orthogonal polynomials and reducer; see theorem (12). Not only does this provide a means to find analytical expressions for the sequence of spectral densities corresponding to the new system-environment interaction after embedding environmental degrees of freedom into the system for the particle and phonon mappings in the gapless spectral densities case (theorem (20) and corollary (86) respectively), but it provides physical meaning to this abstract mathematical construct.

Using convergence theorems of Szegö and deriving the fixed point in the sequence of secondary measures; we have combined these results to obtain a convergence theorem of the sequence of residual spectral densities for the particle and phonon cases, theorem (24) (or equivalently, the sequence of secondary measures, theorem (23)). What is more, because the criterion for convergence (definition 21) is solely in terms of the initial spectral density once the desired chain mapping is chosen, the convergence criterion is readily applicable to a particular problem. Furthermore, we see that any unbounded spectral density will not satisfy this criterion. This is reflected in the sequence generated in the examples section for the case of the family of power-law spectral densities with exponential cut off as the sequence does not converge, section 4.2 .

We give two examples where we can find explicit analytical expressions for the chain coefficients and the sequence of residual spectral densities. These are the family of spectral densities used in the Spin-Boson model, which have spectral densities of the form $x^{s}$ with finite support $I=\left[0, \omega_{c}\right]$ and $x^{s} e^{x / \omega_{c}}$ with semi-infinite support $I=[0,+\infty)$, where $s>-1$. Furthermore, we show how the residual spectral densities of the mapping for both phonon and particle cases are related for different families of the mapping as demonstrated by the identity $\tilde{m}_{0 s}^{1}(x)=\tilde{m}_{0 s / 2}^{0}(x)$ and Eq. (255) and (270). 
We note that when this convergence of the embeddings is achieved (i.e. inequality (231) is satisfied), the part of the chain corresponding to the new environment has a very universal property: all its couplings and frequencies become constant (Corollary 21). This is to say, they are translationally invariant. This suggests a universal way of simulating the environment as all the characteristic features of the environment are now embedded into the system, as discussed for the mapping of [18] in [22. What is not so clear however, is what is the most effective chain mapping for simulating the environment. The general formalism of chain mapping for open quantum systems developed here, paves the way to answering these questions.

\section{Appendix}

6.1 Proof that the relation between the Stieltjes transforms of two consecutive measures is invalid for gapped measures

In the case of a gapless interval $I=[a, b]$, the relation between the Stieltjes transforms of subsequent measures, Eq (21): $S_{\rho}(z)=z-C_{1}(d \mu)-1 / S_{\mu}(z), z \in \mathbb{C}-I$ is well defined because $S_{\mu}(z)$ does not vanish on $\mathbb{C}-I$ :

Proof Let $z=x+i y \quad x, y \in \mathbb{R}$

$$
S_{\mu}(z)=\int_{a}^{b} \frac{\mu(t) d t}{x+i y-t}=\int_{a}^{b} \frac{(x-t) \mu(t) d t}{(x-t)^{2}+y^{2}}-i y \int_{a}^{b} \frac{\mu(t) d t}{(x-t)^{2}+y^{2}}
$$

Hence for $S_{\mu}(z)=0$ we need $y=0$, thus $z=x \in \mathbb{C}-I$, however, under these conditions $(x-t) \mu(t)$ cannot change sign in $t \in I$, therefore $S_{\mu}(z) \neq 0$.

In the case of a gapped interval $I=[a, b] \cup[c, d], S_{\mu}(z)$ the Stieltjes transformation is defined by

$$
S_{\mu}(z)=\int_{a}^{b} \frac{\mu(t) d t}{z-t}+\int_{c}^{d} \frac{\mu(t) d t}{z-t} .
$$

Unfortunately, this expression vanishes on a point in $[b, c]$

Proof Taking real and imaginary parts of $S_{\mu}(z)$ followed by setting the imaginary part to zero, we find

$$
S_{\mu}(x)=\int_{a}^{b} \frac{(x-t) \mu(t) d t}{(x-t)^{2}}+\int_{c}^{d} \frac{(x-t) \mu(t) d t}{(x-t)^{2}},
$$

where $z=x \in \mathbb{R}-I$. However, the numerators of the first and second integrals have a different sign for $x \in[b, c]$. Thus due to the continuity of $S_{\mu}(x)$ in $] b, c[$ and the existence of the limits $\lim _{x \rightarrow b} S_{\mu}(x)=+\infty, \lim _{x \rightarrow c} S_{\mu}(x)=-\infty$, there exists a point $z_{0}$ in $\mathbb{C}-I$ such that $S_{\mu}\left(z_{0}\right)=0$.

Consequently, one cannot define a secondary measure as in definition (8) which satisfy theorem (5), through this relation because the Stieltjes transform must be holomorphic. 
6.2 Methods for calculating the reducer

Theorem 25 If for a measure $d \mu(x)=\bar{\mu}(x) d x, \bar{\mu}(x)$ satisfies a Lipschitz condition over its support interval $[a, b]$, then

$$
\varphi(d \mu ; x)=2 \bar{\mu}(x) \ln \left(\frac{x-a}{b-x}\right)-2 \int_{a}^{b} \frac{\bar{\mu}(t)-\bar{\mu}(x)}{t-x} d t .
$$

Proof From definitions (11) and (7) we have

$$
\varphi(d \mu ; x)=\lim _{\epsilon \rightarrow 0^{+}} 2 \int_{a}^{b} \frac{(x-t) \bar{\mu}(t) d t}{(x-t)^{2}+\epsilon^{2}} .
$$

Writing this as

$$
\begin{aligned}
\varphi(d \mu ; x)= & \lim _{\epsilon \rightarrow 0^{+}} 2 \int_{a}^{b} \frac{(x-t) \bar{\mu}(x)}{(x-t)^{2}+\epsilon^{2}} d t+\lim _{\epsilon \rightarrow 0^{+}} 2 \int_{a}^{b} \frac{(x-t)(\bar{\mu}(t)-\bar{\mu}(x))}{(x-t)^{2}+\epsilon^{2}} d t \\
= & \lim _{\epsilon \rightarrow 0^{+}} 2 \int_{a}^{b} \frac{(x-t) \bar{\mu}(x)}{(x-t)^{2}+\epsilon^{2}} d t-2 \int_{a}^{b} \frac{\bar{\mu}(t)-\bar{\mu}(x)}{t-x} d t \\
& -\lim _{\epsilon \rightarrow 0^{+}} \int_{a}^{b}\left(\frac{2 \epsilon^{2}}{(x-t)^{2}+\epsilon^{2}}\right) \frac{\bar{\mu}(t)-\bar{\mu}(x)}{x-t} d t .
\end{aligned}
$$

1) The first integral in line (288) reduces to

$$
\begin{aligned}
\lim _{\epsilon \rightarrow 0^{+}} 2 \int_{a}^{b} \frac{(x-t) \bar{\mu}(x)}{(x-t)^{2}+\epsilon^{2}} d t & =\lim _{\epsilon \rightarrow 0^{+}} \bar{\mu}(x)\left[\ln \left((a-x)^{2}+\epsilon^{2}\right)-\ln \left((b-x)^{2}+\epsilon^{2}\right)\right] \\
& =2 \bar{\mu}(x) \ln \left(\frac{x-a}{b-x}\right) .
\end{aligned}
$$

2) Now imposing the Lipschitz condition on $\bar{\mu}(x):|\bar{\mu}(x)-\bar{\mu}(t)| \leq K|t-x|$ for some $K$ over interval $[a, b]$, the absolute value of the expression on line (289) reduces to

$$
\begin{aligned}
& \lim _{\epsilon \rightarrow 0^{+}} \int_{a}^{b}\left(\frac{2 \epsilon^{2}}{(x-t)^{2}+\epsilon^{2}}\right) \frac{|\bar{\mu}(t)-\bar{\mu}(x)|}{|t-x|} d t \\
\leq & \lim _{\epsilon \rightarrow 0^{+}} \int_{a}^{b} \frac{2 K \epsilon^{2}}{(x-t)^{2}+\epsilon^{2}} d t \\
= & \lim _{\epsilon \rightarrow 0^{+}} 2 K \epsilon\left[\arctan \left(\frac{b-x}{\epsilon}\right)-\arctan \left(\frac{a-x}{\epsilon}\right)\right] \\
= & 0 .
\end{aligned}
$$

Hence expression on line (289) vanishes.

$\square$

Lemma 11 If $f(x)$ possesses a bounded continuous first derivative on its domain, then it also satisfies a Lipschitz condition on its domain.

Proof See [4]. 
Theorem 26 If for a measure $d \mu(x)=\bar{\mu}(x) d x, \bar{\mu}(x)$ possesses a bounded continuous first derivative on its support interval $I$ and $\bar{\mu}(x)$ can be evaluated on the limits $a, b$, then

$$
\varphi(d \mu ; x)=2\left[\bar{\mu}(a) \ln \left|\frac{x-a}{x}\right|+\bar{\mu}(b) \ln \left|\frac{x}{b-x}\right|+\int_{a}^{b} \bar{\mu}^{\prime}(t) \ln \left|\frac{t-x}{x}\right| d t\right],
$$

where $\bar{\mu}^{\prime}(t)$ is the first derivative of $\bar{\mu}(t)$.

Proof Follows from integrating Eq. (285) by parts and applying lemma (11).

\subsection{Scaling properties of the sequence of beta normalised measures}

Lemma 12 Suppose we have two measures $d \nu^{1}(x)=\bar{\nu}^{1}(x) d x$ and $d \nu^{2}(x)=\bar{\nu}^{2}(x) d x$ with support intervals $I^{1}, I^{2}$ bounded by $\lambda a, \lambda b$ and $a, b$ respectively. If they are related by

$$
\bar{\nu}^{1}(x)=\frac{\bar{\nu}^{2}(x / \lambda)}{\lambda}, \quad \lambda>0
$$

then their sequence of beta normalised measures have the relation

$$
\bar{\nu}_{n}^{1}(x)=\lambda \bar{\nu}_{n}^{2}(x / \lambda) \quad n=1,2,3, \ldots
$$

Proof Using the definition of inner product, definition (2), and relation eq. (297), we find

$$
\langle f, g\rangle_{\bar{\nu}^{2}}=\int_{a}^{b} f(\lambda t) g(\lambda t) d \nu^{1}(t) .
$$

Hence we conclude

$$
P_{n}\left(d \nu^{1} ; x\right)=P_{n}\left(d \nu^{2} ; x / \lambda\right) \quad n=0,1,2, \ldots .
$$

Using this relation and definition ([6), we find

$$
Q_{n}\left(d \nu^{1} ; x\right)=\frac{Q_{n}\left(d \nu^{2} ; x / \lambda\right)}{\lambda} \quad n=0,1,2, \ldots .
$$

Similarly, we have from definition (11)

$$
\varphi\left(d \nu^{1} ; x\right)=\frac{\varphi\left(d \nu^{1} ; x / \lambda\right)}{\lambda} .
$$

Finally, substituting Eq. (300), (301), and (302) into definition (13) we arrive at Eq. (298).

Lemma 13 The sequence of beta normalised measures generated from a measure $d \nu(x)=$ $\bar{\nu}(x) d x$ are invariant under the mapping $d \nu(x) \rightarrow \lambda d \nu(x), \lambda>0$ while keeping the support interval I unchanged.

Proof Follows with a similar line of reasoning as for lemma (12).

Acknowledgements We acknowledge Michael Keyl, Christian Gerard, Sebastian Karl Egger for discussions regarding mathematical technicalities, Nicolas Neumann for pointing out typos and Ramil Nigmatullin for help with latex formatting. This work was supported by the EPSRC CDT on Controlled Quantum Dynamics, the EU STREP project CORNER and the EU Integrating project Q-ESSENCE as well as the Alexander von Humboldt Foundation. 


\section{References}

1. Weiss, U.: Quantum Dissipative Systems, World Scientific, Singapore, 2001

2. Breuer, H.P. and Petruccione, F.:The Theory of Open Quantum Systems, Oxford University Press, 2002

3. Rivas A. and Huelga, S.F.:Open Quantum Systems. An Introduction, Springer Briefs in Physics, Springer, 2011, ISBN: 978-3-642-23353-1

4. Engel, G.S., Calhoun, T.R., Read, E.L., Ahn, T.K., Caronal, T.M., Cheng, Y.C., Blankenship, R.E. and Fleming, G.R.: Evidence for wavelike energy transfer through quantum coherence in photosynthetic systems, Nature 446, 782-786 (2007)

5. Mohseni, M., Rebentrost, P., Lloyd, S. and Aspuru-Guzik, A.: Environment-assisted quantum walks in photosynthetic energy transfer, J. Chem. Phys. 129, 174106 (2008)

6. Plenio, M.B. and Huelga, S.F.: Dephasing-assisted transport: quantum networks and biomolecules, New J. Phys. 10, 113019 (2008)

7. Caruso, F., Chin, A.W., Datta, A., Huelga, S.F. and Plenio, M.B.: Highly efficient energy excitation transfer in light-harvesting complexes: The fundamental role of noise-assisted transport, J. Chem. Phys. 131, 105106 (2009)

8. Proceedings of the 22nd Solvay Conference on Chemistry Quantum Effects in Chemistry and Biology, Procedia Chemistry 3, 1 - 366 (2011)

9. Fleming, G.R., Huelga, S.F. and Plenio, M.B.: Focus on quantum effects and noise in biomolecules, New J. Phys. 13115002 (2011)

10. Leggett, A.J., Chakravarty, S., Dorsey, A.T., Fisher, M.P.A., Garg, A. and Zwerger, W.: Dynamics of the dissipative two-state system, Rev. Mod. Phys. 59, 1-85 (1987)

11. Rivas, A., Huelga, S.F. and Plenio, M.B.: Entanglement and Non-Markovianity of Quantum Evolutions, Phys. Rev. Lett. 105, 050403 (2010)

12. Laine, E.M., Piilo, J. and Breuer, H.P.: Measure for the non-Markovianity of quantum processes, Phy. Rev. A, 81, 062115 (2010)

13. Wolf, M.M., Eisert, J., Cubitt, T.S. and Cirac, J.I.: Assessing non-Markovian dynamics, Phys. Rev. Lett. 101, 150402 (2008)

14. Silbey, R. and Munn, R.W.: General theory of electronic transport in molecular crystals. I. Local linear electron-phonon coupling. J. Chem. Phys. 72, 27632773 (1980)

15. Makri, N.: Numerical path integral techniques for long-time quantum dynamics of dissipative systems, J. Math. Phys. 36, 2430 (1995)

Thorwart M., Eckel, J., Reina, J.H., Nalbach, P., Weiss, S.: Enhanced quantum entanglement in the non-Markovian dynamics of biomolecular excitons, Chem. Phys. Lett. 478, 234 (2009)

16. Ishizaki, A., Tanimura, Y.: Quantum Dynamics of System Strongly Coupled to LowTemperature Colored Noise Bath: Reduced Hierarchy Equations Approach, J. Phys. Soc. Jap. 12, $31313134(2005)$

Ishizaki, A., Fleming, G.R.: Unified treatment of quantum coherent and incoherent hopping dynamics in electronic energy transfer: Reduced hierarchy equation approach, J. Chem. Phys. 130, 234111 (2009)

17. Roden, J., Eisfeld, A., Wolff, W., Strunz, W. T.: Influence of Complex Exciton-Phonon Coupling on Optical Absorption and Energy Transfer of Quantum Aggregates, Phys. Rev. Lett. 103, 058301 (2009)

18. Chin, A.W., Rivas, A., Huelga, S.F. and Plenio, M.B.: Exact mapping between system-reservoir quantum models and semi-infinite discrete chains using orthogonal polynomials, J Math Phys 51, 092109 (2010)

19. Prior, J., Chin, A.W., Huelga, S.F., Plenio, M.B.: Efficient Simulation of Strong SystemEnvironment Interactions, Phys. Rev. Lett. 105, 050404, (2010) 
20. Vojta, M., Tong, N.H., Bulla, R.: Quantum Phase Transitions in the Sub-Ohmic Spin-Boson Model: Failure of the Quantum-Classical Mapping, Phys. Rev. Lett. 94, 070604 (2005)

Bulla, R., Costi, T. and Pruschke, T.: Numerical renormalization group method for quantum impurity systems, Rev. Mod. Phys. 80, 395 (2008)

21. Martinazzo, R., Vacchini, B., Hughes, K.H. and Burghardt, I.: Universal Markovian reduction of brownian particle dynamics, J. Chem. Phys. 134, 011101, (2011)

22. Chin, A.W., Huelga, S.F. and Plenio, M.B.: Chapter 7: Chain representations of open quantum systems and their numerical simulation with time-adapative density matrix renormalisation group methods, To appear in Semiconductors and Semimetals, Volume 85, Elsevier, London, (2012)

23. Martinazzo, R., Hughes, K.H. and Burghardt, I.: Unraveling a Brownian particle's memory with effective mode chains, Phys. Rev. E 84, 030102 (2011)

24. Imamoglu, A.: Stochastic wave-function approach to non-Markovian systems, Phys. Rev. A 50, 3650-3653 (1994)

Garraway, B.M.: Nonperturbative decay of an atomic system in a cavity, Phys. Rev. A 55, 2290$2303(1997)$

Breuer, H.-P.: Genuine quantum trajectories for non-Markovian processes, Phys. Rev. A 70, 012106 (2004)

25. Teschl, G.: Jacobi Operators and Completely Integrable Nonlinear Lattices, Mathematical Surveys and Monographs, Volume 72, Amer. Math. Soc., Providence (2000)

26. John, S.: Strong localization of photons in certain disordered dielectric superlattices, Phys. Rev. Lett. 58, 2486 (1987)

Yablonovitch, E.: Inhibited Spontaneous Emission in Solid-State Physics and Electronics, Phys. Rev. Lett. 58, 2059 (1987)

27. Gautschi, W.: Orthogonal Polynomials, Computation and Approximation, (Numerical Mathematics and Scientific Computation), Oxford Science Publications, ISBN 0-19-850672-4

28. Berg, C.: Annales de la Faculté des Sciences de Toulouse, Sér 6 Vol. S5 (1996), p.932

29. Shermann, J.: On the numerators of the convergents of the Stieltjes continued fractions, Trans.Amer. Math.Soc. 35, 64-87 (1933)

30. Groux, R.: Sur une mesure rendant orthogonaux les polynomes secondaires, C.R Acad.Sci. Paris, Ser.I. 345, 373-376 (2007)

31. Backer, G.A., and Graves-Morris, P.: Pade approximants, Cambridge University Press, (1965)

32. Buchwalter, H., Theorie Spectrale, Cours de DEA universite Claude Bernard Lyon, Chapter 1, (1983)

33. Caldeira, A. and Leggett, A.: Quantum Tunnelling in a Dissipative System, Annals of Physics 149, 374-456 (1983)

34. Berezin, F.A.: The Method of Second Quantization, Academic Press New York San Francisco London, (1966) ISBN 0-12-089450-5

35. Derezinski, J. and Bruneau, L.: Pauli-Fierz Hamiltonians defined as quadratic forms, Rep. Math. Phys 54, 169-199 (2004)

36. Reed, M. and Simon, B.: Methods of Mathematical Physics, Volume 2, sec X.2: Perturbations of self-adjoint operators, ISBN: 0-12-585050-6

37. Nelson, E.: Interation of Nonrelativistic Particles with a Quantized Scalar Field, J. Math. Phys. 59 (1964)

38. Blaizot, J.-P. and Ripka, G.: Quantum Theory of Finite Systems. MIT Press (1985)

39. Leggett, A.J.: Quantum tunneling in the presence of an arbitrary linear dissipation mechanism, Phy. Rev. B. 30, 12081218 (1984)

Garg, A., Onuchic, J.N. and Ambegaokar, V.: Effect of friction on electron transfer in biomolecules, J. Chem. Phys. 83, 4491 (1985)

40. Szegö, G.: Orthogonal Polynomials, American Mathematical Society, Providence, Rhode Island (1975). See chapter 12 
41. Arnold, L.: On Wigner's Semicircle Law for the Eigenvalues of Random Matrices, Journal of Probability theory and related fields, p191-198, Vol 19, issue 3. Springer (1971)

42. Abramowitz, M. and Stegun, I.A.: Handbook of Mathematical Functions with Formulas, Graphs, and Mathematical Tables, New York: Dover. ISBN 0-486-61272-4

43. Adams, R.A. and Fournier, J.F.: Sobolev Spaces, Volume 140, Second Edition, Elservier Science Ltd (2003), ISBN: 0-12-044143-8 